\title{
OGLE-2017-BLG-0406: Spitzer Microlens Parallax Reveals Saturn-mass Planet Orbiting M-dwarf Host in the Inner Galactic Disk
}

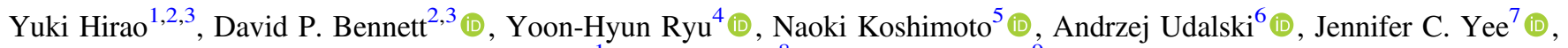
Takahiro Sumi ${ }^{1}$, Ian A. Bond ${ }^{8}$, Yossi Shvartzvald ${ }^{9}$ (i), and

Fumio Abe ${ }^{10}$, Richard K. Barry ${ }^{11}$ (1), Aparna Bhattacharya ${ }^{2,3}$, Martin Donachie ${ }^{12}$, Akihiko Fukui ${ }^{13,14}$, Yoshitaka Itow ${ }^{10}{ }^{(1)}$, Iona Kondo ${ }^{1}(\mathbb{D})$, Man Cheung Alex Li ${ }^{12}$, Yutaka Matsubara ${ }^{10}$, Taro Matsuo ${ }^{1}(\mathbb{0})$, Shota Miyazaki ${ }^{1}$ (1), Yasushi Muraki ${ }^{10}$, Masayuki Nagakane ${ }^{1}$, Clément Ranc ${ }^{2}$ (10), Nicholas J. Rattenbury ${ }^{12}$ (10), Haruno Suematsu ${ }^{1}$, Hiroshi Shibai ${ }^{1}$, Daisuke Suzuki ${ }^{1}$ (D), Paul J. Tristram ${ }^{15}$, Atsunori Yonehara ${ }^{16}$

(The MOA Collaboration),

J. Skowron ${ }^{6}\left(\mathbb{0}\right.$, R. Poleski ${ }^{6,17}$, P. Mróz ${ }^{18}$, M. K. Szymański ${ }^{6}$, I. Soszyński ${ }^{6}$, S. Kozłowski ${ }^{6}$, P. Pietrukowicz ${ }^{6}$ (1), K. Ulaczyk ${ }^{6}$ (1), K. Rybicki ${ }^{6}$, P. Iwanek ${ }^{6}$

(The OGLE Collaboration),

Michael D. Albrow ${ }^{19}$, Sun-Ju Chung ${ }^{4,20}$ (1) , Andrew Gould ${ }^{4,21,22}$, Cheongho Han ${ }^{23}$ (1) , Kyu-Ha Hwang ${ }^{4}$ (1), Youn Kil Jung ${ }^{4}$, In-Gu Shin ${ }^{4}$ (1), Weicheng Zang ${ }^{24}$ (10), Sang-Mok Cha ${ }^{4,25}$, Dong-Jin Kim ${ }^{4}$, Hyoun-Woo Kim ${ }^{4}$, Seung-Lee Kim ${ }^{4,20}$, Chung-Uk Lee ${ }^{4}$, Dong-Joo Lee ${ }^{4}$, Yongseok Lee ${ }^{4,25}$, Byeong-Gon Park ${ }^{4,20}$, Richard W. Pogge ${ }^{22}$

(The KMTNet Collaboration),

Charles A. Beichman ${ }^{26}$, Geoffery Bryden ${ }^{27}$, Sebastiano Calchi Novati ${ }^{26}$, Sean Carey ${ }^{26}$ (1), B. Scott Gaudi ${ }^{22}$, Calen B. Henderson ${ }^{26}\left(\mathbb{D}\right.$, Wei $\mathrm{Zhu}^{28}$

(The Spitzer Team),

Etienne Bachelet ${ }^{29}\left(\mathbb{D}\right.$, Greg Bolt ${ }^{30}$, Grant Christie ${ }^{31}$, Markus Hundertmark ${ }^{32}$, Tim Natusch ${ }^{33,34}$, Dan Maoz ${ }^{35}$, Jennie McCormick ${ }^{36}$, Rachel A. Street ${ }^{29}$ (1) , Thiam-Guan $\operatorname{Tan}^{37}$ (1) , Yiannis Tsapras ${ }^{32}$

(The LCO and $\mu$ FUN Follow-up Teams),

U. G. Jørgensen ${ }^{38}$, M. Dominik ${ }^{39}$, V. Bozza ${ }^{40,41}$ Di , J. Skottfelt ${ }^{42}$, C. Snodgrass ${ }^{43}$, S. Ciceri ${ }^{44}$, R. Figuera Jaimes ${ }^{24,39}$, D. F. Evans ${ }^{45}$, N. Peixinho ${ }^{46}$, T. C. Hinse ${ }^{47}$, M. J. Burgdorf ${ }^{48}$, J. Southworth ${ }^{45}$, S. Rahvar ${ }^{49}$, S. Sajadian ${ }^{50}$, M. Rabus ${ }^{51}$ (1) C. von Essen $^{52}$, Y. I. Fujii ${ }^{38,53}$, J. Campbell-White ${ }^{54}$, S. Lowry ${ }^{54}$, C. Helling ${ }^{39}$, L. Mancini ${ }^{55,56,57,58}$ (D) , L. Haikala ${ }^{59}$

(The MindSTEp Collaboration), and

and

Ryo Kandori ${ }^{60}$

(The IRSF Team)

${ }^{1}$ Department of Earth and Space Science, Graduate School of Science, Osaka University, 1-1 Machikaneyama, Toyonaka, Osaka 560-0043, Japan

${ }^{2}$ Laboratory for Exoplanets and Stellar Astrophysics, NASA/Goddard Space Flight Center, Greenbelt, MD 20771, USA

${ }^{3}$ Department of Astronomy, University of Maryland, College Park, MD 20742, USA

${ }^{4}$ Korea Astronomy and Space Science Institute, Daejon 34055, Republic of Korea

${ }^{5}$ Department of Astronomy, Graduate School of Science, The University of Tokyo, 7-3-1 Hongo, Bunkyo-ku, Tokyo 113-0033, Japan

${ }^{6}$ Astronomical Observatory, University of Warsaw, Al. Ujazdowskie 4, 00-478 Warszawa, Poland

${ }^{7}$ Center for Astrophysics | Harvard \& Smithsonian, 60 Garden Street, Cambridge, MA 02138, USA

${ }^{8}$ Institute of Information and Mathematical Sciences, Massey University, Private Bag 102-904, North Shore Mail Centre, Auckland, New Zealand

${ }^{9}$ Department of Particle Physics and Astrophysics, Weizmann Institute of Science, Rehovot 76100, Israel

${ }^{10}$ Institute for Space-Earth Environmental Research, Nagoya University, Nagoya 464-8601, Japan

${ }^{11}$ Astrophysics Science Division, NASA/Goddard Space Flight Center, Greenbelt, MD 20771, USA

12 Department of Physics, University of Auckland, Private Bag 92019, Auckland, New Zealand

${ }^{13}$ Department of Earth and Planetary Science, Graduate School of Science, The University of Tokyo, 7-3-1 Hongo, Bunkyo-ku, Tokyo 113-0033, Japan

${ }^{14}$ Instituto de Astrofísica de Canarias, Vía Láctea s/n, E-38205 La Laguna, Tenerife, Spain

${ }^{15}$ University of Canterbury, Mount John Observatory, P.O. Box 56, Lake Tekapo 8770, New Zealand

${ }^{16}$ Department of Physics, Faculty of Science, Kyoto Sangyo University, Kyoto 603-8555, Japan

${ }^{17}$ Department of Astronomy, Ohio State University, 140 West 18th Avenue, Columbus, OH 43210, USA

${ }^{18}$ Division of Physics, Mathematics, and Astronomy, California Institute of Technology, Pasadena, CA 91125, USA

${ }^{19}$ University of Canterbury, Department of Physics and Astronomy, Private Bag 4800, Christchurch 8020, New Zealand

${ }^{20}$ Korea University of Science and Technology, Daejeon 34113, Republic of Korea

${ }^{21}$ Max-Planck-Institute for Astronomy, Königstuhl 17, D-69117 Heidelberg, Germany

${ }^{22}$ Department of Astronomy, Ohio State University, 140 W. 18th Avenue, Columbus, OH 43210, USA

${ }^{23}$ Department of Physics, Chungbuk National University, Cheongju 28644, Republic of Korea

${ }^{24}$ Physics Department and Tsinghua Centre for Astrophysics, Tsinghua University, Beijing 100084, People's Republic of China

${ }^{25}$ School of Space Research, Kyung Hee University, Yongin, Kyeonggi 17104, Republic of Korea

${ }^{26}$ IPAC, Mail Code 100-22, Caltech, 1200 E. California Boulevard, Pasadena, CA 91125, USA

${ }^{27}$ Jet Propulsion Laboratory, California Institute of Technology, 4800 Oak Grove Drive, Pasadena, CA 91109, USA

${ }^{28}$ Canadian Institute for Theoretical Astrophysics, University of Toronto, 60 St. George Street, Toronto, ON M5S 3H8, Canada

${ }^{29}$ Las Cumbres Observatory, 6740 Cortona Drive, Suite 102, Goleta, CA 93117, USA

${ }^{30}$ Craigie Observatory, Western Australia, Australia

${ }^{31}$ Auckland Observatory, Auckland, New Zealand

32 Astronomisches Rechen-Institut, Zentrum für Astronomie der Universität Heidelberg (ZAH), D-69120 Heidelberg, Germany

${ }^{33}$ Auckland Observatory, Auckland, New Zealand 
${ }^{34}$ Institute for Radio Astronomy and Space Research (IRASR), AUT University, Auckland, New Zealand

${ }^{35}$ School of Physics and Astronomy, Tel-Aviv University, Tel-Aviv 6997801, Israel

${ }^{36}$ Farm Cove Observatory, Centre for Backyard Astrophysics, Pakuranga, Auckland, New Zealand ${ }^{37}$ Perth Exoplanet Survey Telescope, Perth, Australia

${ }^{38}$ Niels Bohr Institute \& Centre for Star and Planet Formation, University of Copenhagen Øster Voldgade 5, DK-1350—Copenhagen, Denmark

${ }^{39}$ Centre for Exoplanet Science, SUPA School of Physics \& Astronomy, University of St Andrews, North Haugh, St Andrews, KY16 9SS, UK

Dipartimento di Fisica "E.R. Caianiello," Università di Salerno, Via Giovanni Paolo II 132, I-84084, Fisciano, Italy

${ }^{41}$ Istituto Nazionale di Fisica Nucleare, Sezione di Napoli, Napoli, Italy

${ }^{42}$ Centre for Electronic Imaging, Department of Physical Sciences, The Open University, Milton Keynes, MK7 6AA, UK

${ }^{43}$ Institute for Astronomy, University of Edinburgh, Royal Observatory, Edinburgh EH9 3HJ, UK

${ }^{44}$ Department of Astronomy, Stockholm University, AlbaNova University Centre, SE-106 91 Stockholm, Sweden

${ }^{45}$ Astrophysics Group, Keele University, Staffordshire, ST5 5BG, UK

${ }^{46}$ Centro de Astronomía (CITEVA), Universidad de Antofagasta, Avda. U. de Antofagasta 02800, Antofagasta, Chile

${ }^{47}$ Chungnam National University, Department of Astronomy and Space Science, 34134 Daejeon, Republic of Korea

${ }^{48}$ Universität Hamburg, Faculty of Mathematics, Informatics and Natural Sciences, Department of Earth Sciences, Meteorological Institute, Bundesstraße 55, D-20146 Hamburg, Germany

${ }^{49}$ Department of Physics, Sharif University of Technology, P.O. Box 11155-9161 Tehran, Iran

${ }^{50}$ Department of Physics, Isfahan University of Technology, Isfahan, Iran

${ }^{51}$ Instituto de Astrofísica, Pontificia Universidad Católica de Chile, Av. Vicuña Mackenna 4860, 7820436 Macul, Santiago, Chile

${ }^{52}$ Stellar Astrophysics Centre, Department of Physics and Astronomy, Aarhus University, Ny Munkegade 120, DK-8000 Aarhus C, Denmark

${ }_{54}$ Institute for Advanced Research, Nagoya University, Furo-cho, Chikusa-ku, Nagoya, 464-8601, Japan

${ }^{54}$ Centre for Astrophysics \& Planetary Science, The University of Kent, Canterbury CT2 7NH, UK

${ }^{55}$ Dipartimento di Fisica, Universitá di Roma Tor Vergata, Via della Ricerca Scientifica 1, I-00133 Roma, Italy

${ }^{56}$ Max Planck Institute for Astronomy, Königstuhl 17, D-69117 Heidelberg, Germany

${ }^{57}$ INAF-Astrophysical Observatory of Turin, Via Osservatorio 20, I-10025 Pino Torinese, Italy

${ }^{58}$ International Institute for Advanced Scientific Studies (IIASS), Via G. Pellegrino 19, I-84019 Vietri sul Mare (SA), Italy

${ }^{59}$ Universidad de Atacama, Copiapo, Chile

${ }^{60}$ Astrobiology Center of NINS, 2-21-1, Osawa, Mitaka, Tokyo 181-8588, Japan

Received 2020 April 20; revised 2020 June 4; accepted 2020 June 6; published 2020 July 23

\begin{abstract}
We report the discovery and analysis of the planetary microlensing event OGLE-2017-BLG-0406, which was observed both from the ground and by the Spitzer satellite in a solar orbit. At high magnification, the anomaly in the light curve was densely observed by ground-based-survey and follow-up groups, and it was found to be explained by a planetary lens with a planet/host mass ratio of $q=7.0 \times 10^{-4}$ from the light-curve modeling. The ground-only and Spitzer-"only" data each provide very strong one-dimensional (1D) constraints on the $2 \mathrm{D}$ microlens parallax vector $\pi_{\mathrm{E}}$. When combined, these yield a precise measurement of $\pi_{\mathrm{E}}$ and of the masses of the host $M_{\text {host }}=0.56 \pm 0.07 M_{\odot}$ and planet $M_{\text {planet }}=0.41 \pm 0.05 M_{\text {Jup }}$. The system lies at a distance $D_{\mathrm{L}}=5.2 \pm 0.5 \mathrm{kpc}$ from the Sun toward the Galactic bulge, and the host is more likely to be a disk population star according to the kinematics of the lens. The projected separation of the planet from the host is $a_{\perp}=3.5 \pm 0.3$ au (i.e., just over twice the snow line). The Galactic-disk kinematics are established in part from a precise measurement of the source proper motion based on OGLE-IV data. By contrast, the Gaia proper-motion measurement of the source suffers from a catastrophic $10 \sigma$ error.
\end{abstract}

Unified Astronomy Thesaurus concepts: Gravitational microlensing (672); Gravitational microlensing exoplanet detection (2147)

Supporting material: data behind figure

\section{Introduction}

Gravitational microlensing has a unique strength in its sensitivity to planets with masses as low as Earth mass (Bennett \& Rhie 1996) just beyond the snow line (Gould \& Loeb 1992), where the core accretion theory of planetary formation predicts the most efficient planet formation (Ida \& Lin 2005). Because it does not rely on the light from the host star, microlensing can detect the planets orbiting around faint stars like M-dwarfs and brown dwarfs, and can even detect free-floating planets (Sumi et al. 2011; Mróz et al. 2017, 2018, 2019, 2020). Microlensing can also detect planets in the Galactic bulge because microlensing events can be caused by stars at any distance between Earth and the Galactic bulge, where most of the stars that act as sources lie. This is complementary to other planet detection techniques, such as the radial velocity (Butler et al. 2006) and transit (Borucki et al. 2011) methods, which are most sensitive to planets in short period orbits. Therefore, the microlensing method is essential for the complete demographic census of Galactic planetary systems (Gaudi 2012; Tsapras 2018).
Several statistical studies based on the discovered microlensing planets have been conducted and revealed the planet occurrence rates beyond the snow line (Gould et al. 2010; Sumi et al. 2010; Cassan et al. 2012; Shvartzvald et al. 2016) and the possible paucity of planets in the Galactic bulge (Penny et al. 2016). One of the most important microlensing statistical results is that of Suzuki et al. (2016), who found a clear break and likely peak in the planet-host mass ratio function at a mass ratio of $q \sim 10^{-4}$ using 30 exoplanets detected by microlensing. This peak was confirmed by Udalski et al. (2018) and Jung et al. (2018), who determined that the peak occured at a mass ratio of $q \approx 6 \times 10^{-5}$. A comparison of the Suzuki et al. (2016) results to population synthesis models based on the core accretion theory (Suzuki et al. 2018) reveals a discrepancy between the smooth mass ratio distribution for the microlens planets and the predicted deficit of planets, with mass ratios lying in the range of $10^{-4}<q<4 \times 10^{-4}$. This predicted gap in the mass ratio distribution (Ida \& Lin 2004) is due to the runaway gas accretion process (Pollack et al. 1996; 
Lissauer et al. 2009), which has long been considered a fundamental aspect of the core accretion theory. So, the microlensing results seem to imply that a major change in the theory is needed. In fact, recent three-dimensional highresolution numerical calculations (Szulágyi et al. 2014; J. Szulágyi et al. 2020, in preparation) indicate that runaway gas accretion often halted or decreased due to the circumplanetary disk formation and suggest that earlier, lower resolution three-dimensional calculations had numerical artifacts that favored the runaway gas accretion scenario. Comparison of the population synthesis results to ALMA protoplanetary disk observations also support this conclusion (Nayakshin et al. 2019).

Microlensing light-curve models provide the lens planet-host mass ratios, but they do not usually provide the lens mass and distance. To measure the properties of lens systems, one needs additional observables that yields mass-distance relation of the lens systems, such as the angular Einstein ring radius $\theta_{\mathrm{E}}$ and the microlens parallax $\pi_{\mathrm{E}}$. The measurement of the $\theta_{\mathrm{E}}$ or $\pi_{\mathrm{E}}$ values yields the following mass-distance relations,

$$
M_{\mathrm{L}}=\frac{c^{2}}{4 G} \theta_{\mathrm{E}}^{2} \frac{D_{\mathrm{S}} D_{\mathrm{L}}}{D_{\mathrm{S}}-D_{\mathrm{L}}}=\frac{c^{2}}{4 G} \frac{\mathrm{au}}{\pi_{\mathrm{E}}^{2}} \frac{D_{\mathrm{S}}-D_{\mathrm{L}}}{D_{\mathrm{S}} D_{\mathrm{L}}},
$$

where $D_{\mathrm{L}}$ is the lens distance and the source distance, $D_{\mathrm{S}}$, is known (approximately). If the apparent $K$-band magnitude of the lens star $K_{\mathrm{L} \text {,meas }}$ is measured, then we have a mass-distance relation given by $K_{\mathrm{L} \text {,meas }}=5 \log _{10}\left(D_{\mathrm{L}} / 10 \mathrm{pc}\right)+A_{K}\left(D_{\mathrm{L}}\right)+$ $K_{\text {abs, meas }}\left(M_{\mathrm{L}}\right)$, where $K_{\text {abs, meas }}\left(M_{\mathrm{L}}\right)$ is a $K$-band mass-luminosity relation and $A_{K}\left(D_{\mathrm{L}}\right)$ is a model of the extinction in the foreground of the lens star. Measurements of the lens brightness in other passbands yield independent mass-distance relations. Combining any two of these mass-distance relations will yield the lens mass $M_{\mathrm{L}}$ and distance $D_{\mathrm{L}}$. The most elegant solution is obtained if both the angular Einstein radius, $\theta_{\mathrm{E}}$, and the microlensing parallax, $\pi_{\mathrm{E}}$, are measured because this distance dependence cancels, enabling unique determinations of $M_{\mathrm{L}}$ and $D_{\mathrm{L}}$ by the following relations,

$$
M_{\mathrm{L}}=\frac{\theta_{\mathrm{E}}}{\kappa \pi_{\mathrm{E}}} ; D_{\mathrm{L}}=\frac{\mathrm{au}}{\pi_{\mathrm{E}} \theta_{\mathrm{E}}+\pi_{\mathrm{S}}},
$$

where $\kappa=4 G /\left(c^{2} \mathrm{au}\right)=8.1439 \mathrm{mas} / M_{\odot}$ and $\pi_{\mathrm{S}}=1 \mathrm{au} / D_{\mathrm{S}}$ (Gould 1992, 2000). For binary events, $\theta_{\mathrm{E}}$ can be routinely measured by the source radius crossing time, $t_{*}$, provided that the source crosses a caustic curve or closely approaches to a caustic cusp. This gives $\theta_{\mathrm{E}}=\theta_{*} t_{\mathrm{E}} / t_{*}$, where $\theta_{\text {ast }}$ is the angular radius of the source, which can be determined from the lightcurve model values for the source brightness and color (Albrow et al. 1998; Yoo et al. 2004).

It can be challenging to obtain the measurements necessary for the other mass-distance relations, aside from the $\theta_{\mathrm{E}}$ relation. Detecting the host star is nearly impossible for bright source stars, and a unique identification of the host star can be difficult if the source star is bright (i.e., a giant star) or if the relative lens-source proper motion is not big enough to resolve the lens and source (Bhattacharya et al. 2017; Koshimoto et al. 2017, 2020). Because the lens-source separation increases as time passes after an event, there are an increasing number of planetary events with mass measurements from host star brightness measurements (Bennett et al. 2006, 2015, 2020; Batista et al. 2015; Bhattacharya et al. 2018; Vandorou et al. 2019), and this is the method that is expected to make most of the exoplanet mass measurements for WFIRST (Bennett \& Rhie 2002; Bennett et al. 2007; Spergel et al. 2015).

The microlensing parallax effect has traditionally been measured due to the effects of the orbital motion of Earth. Dong et al. (2009) made the first such measurement on OGLE2005-BLG-071 (only the second planet detected by microlensing; Udalski et al. 2005), which was made possible in part by the exceptionally large parallax. ${ }^{61}$ However, in general, this annual parallax effect can only be measured for a subset of planetary microlensing events: events that have long durations, like OGLE-2006-BLG-109 (Gaudi et al. 2008; Bennett et al. 2010) and OGLE-2007-BLG-349 (Bennett et al. 2016), have bright source stars and moderately long durations, like MOA2009-BLG-266 (Muraki et al. 2011) and OGLE-2012-BLG0265 (Skowron et al. 2015), or have very special lens-source geometries, such as MOA-2013-BLG-605 (Sumi et al. 2016) and OGLE-2013-BLG-0341 (Gould et al. 2014).

However, $\pi_{\mathrm{E}}$ can also be measured by simultaneously observing lensing events from two well-separated ( au) observatories (Refsdal 1966). Since 2014, almost 1000 events, including both single and binary events, were simultaneously observed from the ground and the Spitzer Space Telescope (Yee et al. 2015a; Zhu et al. 2017). Spitzer observations helped determine the distance to the lens for more than a hundred of those events. To date, ten planetary events were observed by Spitzer. Seven of these are located in the Galactic disk: OGLE2014-BLG-0124 (Udalski et al. 2015b; Beaulieu et al. 2018), OGLE-2015-BLG-0966 (Street et al. 2016), OGLE-2017BLG-1140 (Calchi Novati et al. 2018), OGLE-2016-BLG1067 (Calchi Novati et al. 2019), OGLE-2016-BLG-1195 (Bond et al. 2017; Shvartzvald et al. 2017), KMT-2018-BLG0029 (Gould et al. 2020), and Kojima-1 (Nucita et al. 2018; Fukui et al. 2019; Zang et al. 2020). The lens systems for events OGLE-2016-BLG-1190 (Ryu et al. 2017), OGLE-2018BLG-0596 (Jung et al. 2019), and OGLE-2018-BLG-0799 (W. Zang et al. 2020, in preparation) are reported to be in the Galactic bulge. While observations from Spitzer make it easier to measure the small $\pi_{\mathrm{E}}$ values for bulge lens systems, this ability is undermined by the requirement that events should be discovered at least $\sim 1$ week before Spitzer observations can be requested (Figure 1 from Udalski et al. 2015b). This, combined with the limited 40 day Spitzer observing window for bulge events, leads to incomplete light curves, which can make parallax measurements difficult.

In this paper, we report the discovery and analysis of the planetary microlensing event OGLE-2017-BLG-0406, which was observed both from the ground and in space using the Spitzer telescope. The anomaly in the light curve was well covered by ground-based observations. The additional Spitzer data constrained the parallax parameters-hence the mass and the distance of the lens systems. We describe the ground-based and space-based observations in Section 2 and the data reductions in Section 3. In Section 4, we describe our lightcurve modeling conducted for the ground-based data. We present our Spitzer parallax analysis in Section 5. In Sections 6 to 8 , we present the determinations of source properties and lens properties. Finally, we discuss and summarize the results in Section 9.

\footnotetext{
61 Bennett et al. (2020) confirmed this first planet-event parallax measurement and found a $2 \sigma$ correction, using high-resolution imaging
} 


\section{Observations}

\subsection{Ground-based Observation}

The microlensing event OGLE-2017-BLG-0406 was first discovered on March $27\left(\mathrm{HJD}^{\prime}=\mathrm{HJD}-2,450,000=7839\right)$ by the Optical Gravitational Lensing Experiment (OGLE) collaboration at (R.A., decl. $)(\mathrm{J} 2000)=\left(17^{\mathrm{h}} 55^{\mathrm{m}} 59\right.$ s.92, $\left.-29^{\circ} 51^{\prime} 47^{\prime \prime} \cdot 3\right)$ or $(l, b)=\left(0.3601,-2^{\circ} .4164\right)$ in Galactic coordinates, as alerted by the OGLE Early Warning System (Udalski 2003). The event lies in the OGLE-IV field BLG506, and the observations were conducted at the cadence of once per hour by using the $1.3 \mathrm{~m}$ Warsaw telescope located at Las Campanas Observatory in Chile, equipped with a $1.4 \mathrm{deg}^{2}$ field-of-view CCD camera. The Microlensing Observations in Astrophysics (MOA) group independently discovered this event on May 5 $\left(\mathrm{HJD}^{\prime}=7879\right)$ by using the MOA alert system (Bond et al. 2001) and identified it as MOA-2017-BLG-233. MOA observed this event with 15 minutes cadence by using MOAII telescope at Mt. John University Observatory in New Zealand, equipped with $2.2 \mathrm{deg}^{2}$ field-of-view camera MOAcamIII (Sako et al. 2008). Most observations were conducted in the customized MOA-Red wide band, which is the sum of the standard Cousins $R$ and $I$ bands with occasional observations in the Johnson $V$ band. The event was also independently discovered as KMT-2017-BLG-0243 by the Korean Microlensing Network (KMTNet: Kim et al. 2016) survey using its postseason event finder (Kim et al. 2018). KMTNet observes toward the Galactic bulge by using three $1.6 \mathrm{~m}$ telescopes equipped with $4 \mathrm{deg}^{2}$ camera at the Cerro Tololo InterAmerican Observatory in Chile (CTIO: KMT-C), the South African Astronomical Observatory in South Africa (SAAO: KMT-S), and the Siding Spring Observatory in Australia (SSO: KMT-A). Because this event was in an overlapping region between two fields (KMTNet BLG02 and BLG42), the observations were conducted at a 15 minute cadence.

On June $2\left(\mathrm{HJD}^{\prime}=7907\right)$, the Microlensing Follow-up Network $(\mu$ FUN) collaboration issued an alert that the event was peaking at a high magnification, which means that there is a high probability that the light curve will show an anomaly if the lens star hosts a planet (Griest \& Safizadeh 1998). After the alert, $\mu$ FUN, the Microlensing Network for the Detection of Small Terrestrial Exoplanet (MiNDSTEp) collaboration and Las Cumbres Observatory (LCO) global network of telescope collaboration started high-cadence follow-up observations. $\mu$ FUN used the following telescopes: the $1.3 \mathrm{~m}$ CTIO telescope in Chile, the $0.41 \mathrm{~m}$ Auckland telescope and the $0.36 \mathrm{~m}$ Farm Cove telescope in New Zealand, and the $0.30 \mathrm{~m}$ Perth Exoplanet Survey Telescope (PEST), and the $0.25 \mathrm{~m}$ Craigie telescope in Australia. MiNDSTEp used the $1.54 \mathrm{~m}$ Danish Telescope at La Silla Observatory in Chile. LCO used the $1.0 \mathrm{~m}$ telescopes at CTIO in Chile and at SSO in Australia. Figure 1 shows the light curve of the event.

On June $4\left(\mathrm{HJD}^{\prime}=7909\right)$, deviations from a single-lens fit were noticed just after the peak by the MOA observer. Then the first planetary model was circulated by V. Bozza, and it was confirmed by several modelers. Because the event was very bright ( $\sim 12.5$ mag in $I$ band), some images taken with normal exposure time by survey telescopes were saturated.

We also obtained three near-infrared images taken at different epochs (HJD' $\sim 7911,7918$ and 7942). The observations were made with SIRIUS, a simultaneous imager in $J, H$, and $K_{S}$ bands, covering an area $7.7 \times 7.7 \operatorname{arcmin}^{2}$ with a pixel scale of 0." 45 (Nagayama et al. 2003) on the $1.4 \mathrm{~m}$ InfraRed Survey Facility (IRSF) telescope at SAAO. The observations were conducted to measure the source color rather than for light-curve modeling. The data sets are listed in Table 1.

\subsection{Space-based Observation}

OGLE-2017-BLG-0406 was observed by the Spitzer space telescope with the $3.6 \mu \mathrm{m}$ ( $L$-band) channel of the IRAC camera. Spitzer started to observe this event on June 26 $\left(\mathrm{HJD}^{\prime}=7931\right)$, which was about 3 weeks after the peak because this was the first date that the target was visible in the Spitzer image. This event was chosen for Spitzer observations as part of a long-term (2014-2019) program, according to the protocols of Yee et al. (2015a). Specifically, it met objective criteria defined by Yee et al. (2015a), which meant that it had to be chosen for observations and observed at a specified cadence, independent of whether it had a planet or not. Accordingly, it was observed approximately once per day for the first 4 weeks, but not the final 2 weeks of the program in 2017.

In 2019 (i.e., the final Spitzer microlensing season), essentially all planetary events from 2014 to 2018 were observed for about a week at baseline, primarily to check for systematics in the light curves, in part because of concerns raised by Koshimoto \& Bennett (2019). See Gould et al. (2020) for further discussion. OGLE-2017-BLG-0406 was observed seven times under this program, meaning that there are a total of 28 data points. As discussed in Section 9.1, these seven points must be excluded when determining whether OGLE2017-BLG-0406Lb can enter the Spitzer-statistical sample. For the role of these data in the analysis of systematic effects, see the Appendix.

\section{Data Reduction}

The great majority of the ground-based data were reduced using the pipelines developed by the individual collaborations based on difference image analysis (DIA) method developed by Tomaney \& Crotts (1996) and Alard \& Lupton (1998). The OGLE I-band data were reduced by the OGLE DIA (Woźniak 2000) photometry pipeline (Udalski et al. 2015a). The MOA-Red and $V$-band data were reduced by the MOA DIA pipeline (Bond et al. 2001). KMTNet-I-band data were reduced with their pySIS photometry pipeline (Albrow et al. 2009). $\mu \mathrm{FUN}$ data were reduced using DoPhot (Schechter et al. 1993), and LCO data were reduced using pySIS (Albrow et al. 2009). Danish data were reduced using an updated version of DanDIA (Bramich 2008). IRSF images were reduced using the standard IRSF pipeline and MOA DIA pipeline. Spitzer $L$-band data was reduced using methods described in Calchi Novati et al. (2015).

The error bars must be renormalized to accurately estimate the uncertainties. We use the following formula to rescale the errors, $\sigma^{\prime}{ }_{i}=k \sqrt{\sigma_{i}^{2}+e_{\min }^{2}}$, where $\sigma_{i}$ and $\sigma^{\prime}{ }_{i}$ are original and renormalized error bars in magnitudes, and $k$ and $e_{\min }$ are rescaling factors (Bennett et al. 2008). The value of $e_{\min }$ represents systematic errors that dominate at high magnification or when the target is very bright. First, we fit all the light curves to find a tentative best-fit model. Then we apply $e_{\min }=$ 0.003257 and choose $k$ values to give $\chi^{2} /$ dof $=1$ for a preliminary best-fit model. Finally, all the normalized light curves are fit again and we get the final best-model. In this 

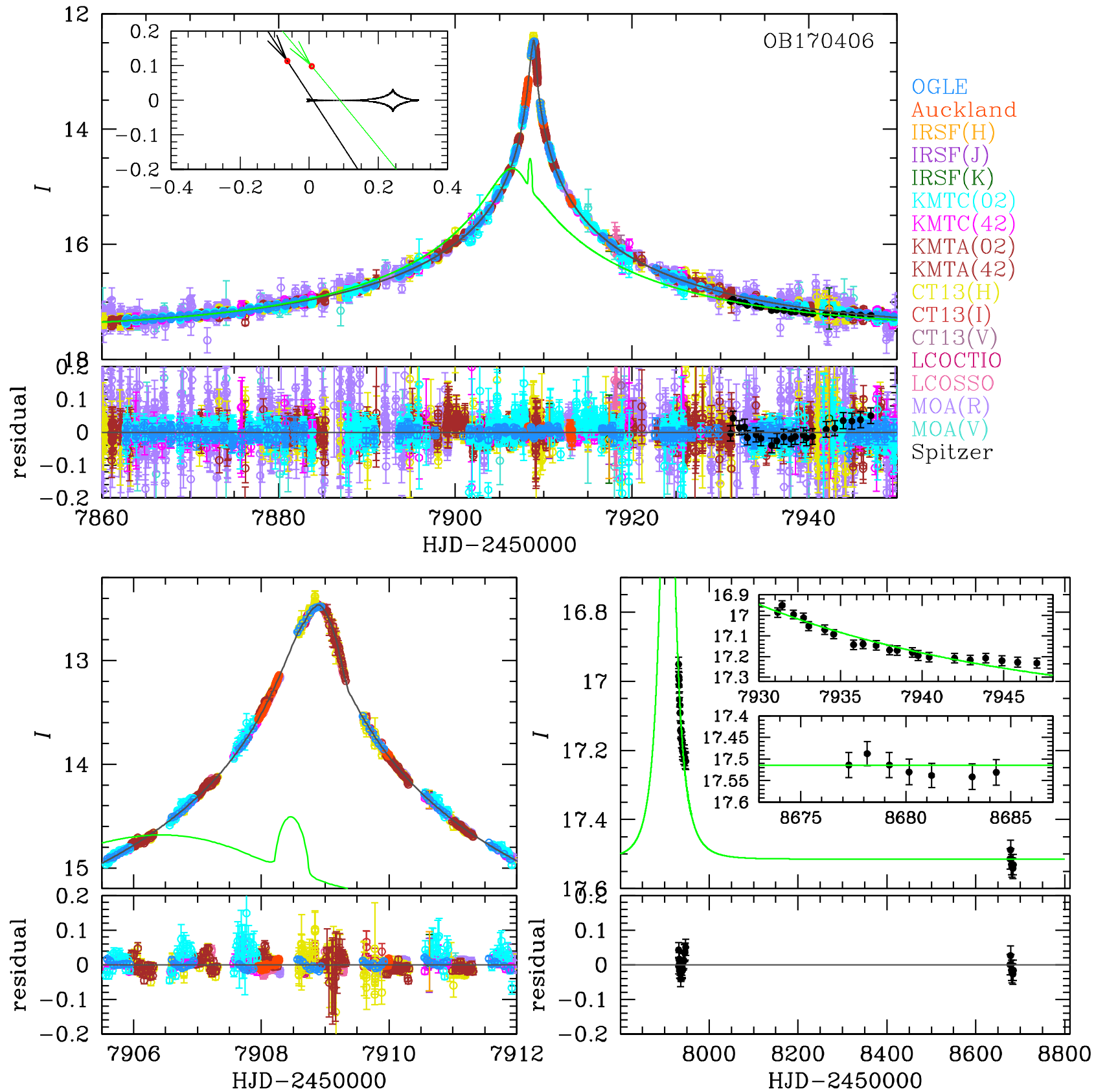

Figure 1. Observed light curve of OGLE-2017-BLG-0406 and best-fit model light curve for the Wide $(+,+)$ model. Data points from different collaborations are shown with different colors. The blue and green solid lines are the model light curves for the ground and Spitzer observations. The bottom left and right panels show a close-up of the anomaly and Spitzer observations with the residuals from the best-fit model, respectively. The insert on the top panel shows the caustic structure.

(The data used to create this figure are available.)

process, we find that there are systematics in the data from Danish, Craigie, Farm Cove, and PEST. Also, Danish data are not consistent with the OGLE and LCO-CTIO data. Hence, they are not used for the analysis. We note that the data from KMT-S are not used because of the systematics that mimic the parallax signal, as described in Section 4. The data sets we used, together with the values of $k$ and $e_{\min }$, are shown in Table 1. We also note that for the OGLE-IV data, we check the standard error correction procedure described in Skowron et al. (2016a) and find that the resulting error bars are similar to those estimated in this work. We choose $k=1$ and $e_{\min }=0$ for IRSF data.

\section{Ground-based Light-curve Analysis}

For the point-source point-lens model, one needs three parameters to characterize the microlens light curve: $t_{0}$, the time of closest approach of the source to the lens mass; $u_{0}$, the impact parameter in units of the angular Einstein radius $\theta_{\mathrm{E}}$; and $t_{\mathrm{E}}$, the Einstein radius crossing time. For the binary-lens model, one needs three additional parameters: $q$, the planet/host mass ratio; $s$, the projected planet-star separation in units of the Einstein radius; and $\alpha$, the angle of the source trajectory relative to the binary-lens axis. When we take account of the finite-source effect and the parallax effect, the angular radius of the source star in units of $\theta_{\mathrm{E}}, \rho$, and the north and east 
Table 1

The Data Sets Used to Model the OGLE-2017-BLG-0406 Light Curve and the Error Correction Parameters

\begin{tabular}{lcccc}
\hline \hline Telescope & Filter & $N_{\text {use }} / N_{\text {obs }}$ & $k$ & $e_{\min }$ \\
\hline OGLE & $I$ & $6185 / 6185$ & 1.441 & 0.003257 \\
MOA & Red & $14611 / 14611$ & 1.970 & 0.003257 \\
& $V$ & $315 / 315$ & 1.794 & 0.003257 \\
KMT-C02 & $I$ & $1558 / 1558$ & 2.537 & 0.003257 \\
KMT-C42 & $I$ & $1713 / 1713$ & 1.897 & 0.003257 \\
KMT-A02 & $I$ & $1903 / 1903$ & 2.590 & 0.003257 \\
KMT-A42 & $I$ & $2068 / 2068$ & 2.550 & 0.003257 \\
KMT-S02 & $I$ & $0 / 2485$ & & \\
KMT-S42 & $I$ & $0 / 2481$ & & \\
Danish & $I$ & $0 / 200$ & & \\
LCO-CTIO & $I$ & $296 / 296$ & 1.323 & 0.003257 \\
LCO-SSO & $I$ & $464 / 464$ & 1.644 & 0.003257 \\
Auckland & $R$ & $82 / 82$ & 2.280 & 0.003257 \\
Craigie & clear & $0 / 677$ & & \\
Farm Cove & clear & $0 / 31$ & & \\
PEST & clear & $0 / 262$ & & \\
CTIO & $I$ & $21 / 21$ & 0.860 & 0.003257 \\
& $V$ & $7 / 7$ & 0.760 & 0.003257 \\
IRSF & $H$ & $92 / 93$ & 1.700 & 0.003257 \\
& $J$ & $3 / 3$ & 1.000 & 0.000 \\
Spitzer & $H$ & $3 / 3$ & 1.000 & 0.000 \\
\hline & $L$ & $3 / 3$ & 1.000 & 0.000 \\
& & $21 / 21$ & 3.120 & 0.003257 \\
\hline
\end{tabular}

components of the microlensing parallax vector, $\pi_{\mathrm{E}, N}$ and $\pi_{\mathrm{E}, E}$, are added for each case. The model light curve is given by

$$
F(t)=A(t) F_{\mathrm{S}, i}+F_{\mathrm{b}, i}
$$

where $F(t)$ is the flux at time $t, A(t)$ is the magnification of the source star at $t$, and $F_{\mathrm{S}, i}$ and $F_{\mathrm{b}, i}$ are the baseline fluxes from the source and blend stars for each data set, $i$, respectively.

We use linear limb-darkening models for the source star. The effective temperature of the source star estimated from the extinction-corrected source color, $(V-I)_{\mathrm{S}, 0}=1.02$, as described in Section 6, is $T_{\text {eff }} \sim 4848 \mathrm{~K}$ (González Hernández \& Bonifacio et al. 2009). Rounding to the nearest $T_{\text {eff }}$ given in Claret (2000) and assuming surface gravity $\log \left[\mathrm{g} /\left(\mathrm{cm} \mathrm{s}^{-2}\right)\right]=4.5$, and metallicity $\log [\mathrm{M} / \mathrm{H}]=0$, we selected limb-darkening coefficients $u_{\lambda}$ to be $u_{I}=0.6049, u_{\mathrm{Red}}=0.6534, u_{V}=0.7796, u_{R}=0.7081, u_{J}=$ $0.4896, u_{H}=0.4252$, and $u_{K_{S}}=0.3642$, respectively (Claret 2000). The MOA-Red value is the mean of the $R$ - and $I$-band values.

We first conduct the light-curve modeling by only using ground-based data. Our light-curve modeling was done using the image-centered ray-shooting method (Bennett \& Rhie 1996; Bennett 2010) and the Markov Chain Monte Carlo (MCMC) algorithm (Verde et al. 2003). Note that the source and blend flux parameters are not MCMC parameters, but are fit linearly to each model following Rhie et al. (1999). To find the global best-fit model, we first conduct a grid search by fixing three parameters $(q, s, \alpha)$ while the other parameters $\left(t_{0}, t_{\mathrm{E}}, u_{0}, \rho\right)$ are allowed to be free. Next, we search for the best-fit model by refining all parameters for those models with the 100 smallest $\chi^{2}$ values as initial parameters. From this modeling, we find a planetary model that has the best-fit values of $q \sim 0.0007$ and $s \sim 1.128$. The best-fit parameters are shown in Table 2 .
Table 2

Standard Models

\begin{tabular}{lcc}
\hline \hline Parameters & Wide $(s>1)$ & Close $(s<1)$ \\
\hline$\chi^{2} /$ dof & $29272.22 / 29285$ & $29325.836 / 29285$ \\
$t_{0}\left(\mathrm{HJD}^{\prime}\right)$ & $7908.809 \pm 0.001$ & $7908.810 \pm 0.001$ \\
$u_{0}\left(10^{-3}\right)$ & $9.437 \pm 0.028$ & $9.454 \pm 0.029$ \\
$t_{\mathrm{E}}($ days $)$ & $37.043 \pm 0.082$ & $36.994 \pm 0.083$ \\
$s$ & $1.129 \pm 0.001$ & $0.895 \pm 0.001$ \\
$q\left(10^{-4}\right)$ & $7.024 \pm 0.090$ & $6.876 \pm 0.091$ \\
$\alpha(\mathrm{rad})$ & $0.993 \pm 0.001$ & $0.993 \pm 0.002$ \\
$\rho\left(10^{-3}\right)$ & $5.861 \pm 0.025$ & $5.830 \pm 0.025$ \\
$f_{S}($ OGLE $)$ & $1.462 \pm 0.004$ & $1.464 \pm 0.004$ \\
$f_{B}($ OGLE $)$ & $0.102 \pm 0.004$ & $0.100 \pm 0.004$ \\
$t_{\text {ast }}($ days $)$ & $0.217 \pm 0.001$ & $0.216 \pm 0.001$ \\
\hline
\end{tabular}

Note. $t_{\text {ast }} \equiv \rho t_{\mathrm{E}}$ is a derived quantity and is not fitted independently. All fluxes are on an 18th mag scale (e.g., $I_{s}=18-2.5 \log \left(f_{s}\right)$ ).

Because the source crosses the central caustic very close to the lens host star as seen in Figure 1, the finite-source effect is well measured. The $\Delta \chi^{2}$ between the best-fit model and the singlelens model is more than 20,000. Thus, the planetary signal is detected confidently. We also explore the binary-source singlelens model (Gaudi 1998) and find that the model is ruled out by $\Delta \chi^{2}>3000$.

High-magnification planetary microlensing events often have a so-called close-wide degeneracy because the structures near the central caustic are very similar to each other for $s \leftrightarrow s^{-1}$, particularly for $s \ll 1$ and $s \gg 1$ (Griest \& Safizadeh 1998; Dominik 1999; Chung et al. 2005). We search for the model with $s<1$ and find the model that has the best-fit values of $q \sim 0.0007$ and $s \sim 0.895$. But this close model has worse $\chi^{2}$ compared to the best-fit wide model by $\Delta \chi^{2} \sim 41$. This difference mostly comes from the data near the peak. Thus, we exclude the close model because of its poor fit.

When $t_{\mathrm{E}}$ is large, we have a chance to measure the orbital parallax effect, which is caused by the acceleration of Earth's orbital motion (Gould 1992; Alcock et al. 1995). We do not expect a significant orbital microlensing parallax signal for such a short event, in the middle of the season, because the acceleration of Earth projected to the bulge is the smallest. We begin by doing a parallax fit without the Spitzer data to independently assess parallax constraints coming from the ground-based data. We conduct the parallax fit by adding the two additional parameters, $\pi_{\mathrm{E}, \mathrm{N}}$ and $\pi_{\mathrm{E}, \mathrm{E}}$. In the first iteration, we found a model with a large $\pi_{\mathrm{E}}$ value of $\sim 0.4$. However, the $\Delta \chi^{2}$ between the standard model and the parallax model comes mostly from KMT-S data, and it was not consistent with the other data sets. Also, the differences were from the baseline. Hence, we conduct parallax analysis without the KMT-S data set because we think that there is a systematic error in the data set, which mimics the deviation caused by the parallax effect. Then we tried the parallax fit again and obtained a smaller $\pi_{\mathrm{E}}$ value of $\sim 0.2$. The best-fit parameters are shown in Table 3 . While the improvement in $\chi^{2}$ is relatively small $\left(\Delta \chi^{2}=6.9\right)$, Figures 2 and 3 show that there is a strong one-dimensional (1D) parallax constraint, which arises from the asymmetry in the light curve induced by the instantaneous acceleration of Earth around the peak of the event (Gould et al. 1994). The relatively small $\Delta \chi^{2}$ simply reflects the fact that this $1 \mathrm{D}$ 
Table 3

Parallax Models for Ground-only Data

\begin{tabular}{lcccc}
\hline \hline Parameters & Wide $(+)$ & Wide $(-)$ & Close $(+)$ & Close $(-)$ \\
\hline$\chi^{2} /$ dof & $29272.225 / 29283$ & $29273.381 / 29283$ & $29318.530 / 29283$ & $29318.828 / 29283$ \\
$t_{0}\left(\mathrm{HJD}^{\prime}\right)$ & $7908.809 \pm 0.001$ & $7908.809 \pm 0.001$ & $7908.809 \pm 0.001$ & $7908.809 \pm 0.001$ \\
$u_{0}\left(10^{-3}\right)$ & $9.402 \pm 0.032$ & $-9.447 \pm 0.030$ & $9.432 \pm 0.027$ & $-9.419 \pm 0.030$ \\
$t_{\mathrm{E}}($ days $)$ & $37.168 \pm 0.096$ & $37.042 \pm 0.091$ & $37.075 \pm 0.076$ & $0.895 \pm 0.001$ \\
$s$ & $1.129 \pm 0.001$ & $1.130 \pm 0.001$ & $6.894 \pm 0.089$ & $0.895 \pm 0.001$ \\
$q\left(10^{-4}\right)$ & $6.972 \pm 0.093$ & $7.073 \pm 0.092$ & $0.993 \pm 0.002$ & $-0.831 \pm 0.090$ \\
$\alpha(\mathrm{rad})$ & $0.991 \pm 0.001$ & $-0.992 \pm 0.002$ & $5.822 \pm 0.025$ & $5.809 \pm 0.025$ \\
$\rho\left(10^{-3}\right)$ & $5.832 \pm 0.025$ & $5.874 \pm 0.025$ & $0.137(0.121) \pm 0.031$ & $0.198(0.168) \pm 0.065$ \\
$\pi_{\mathrm{E}, N}$ & $0.179(0.167) \pm 0.094$ & $0.157(0.168) \pm 0.066$ & $0.064(0.070) \pm 0.014$ & $0.098(0.086) \pm 0.027$ \\
$\pi_{\mathrm{E}, E}$ & $0.097(0.089) \pm 0.037$ & $0.097(0.088) \pm 0.026$ & $0.151(0.141) \pm 0.030$ & $0.221(0.189) \pm 0.068$ \\
$\pi_{\mathrm{E}}$ & $0.204(0.193) \pm 0.093$ & $0.185(0.191) \pm 0.069$ & $0.438(0.538) \pm 0.124$ & $0.459(0.498) \pm 0.155$ \\
$\phi_{\pi}$ & $0.494(0.554) \pm 0.481$ & $0.554(0.507) \pm 0.123$ & $1.460 \pm 0.004$ & $1.459 \pm 0.004$ \\
$f_{S}(\mathrm{CTIO})$ & $1.457 \pm 0.004$ & $1.463 \pm 0.004$ & $0.104 \pm 0.003$ & $0.105 \pm 0.004$ \\
$f_{B}(\mathrm{CTIO})$ & $0.107 \pm 0.004$ & $0.101 \pm 0.004$ & $0.216 \pm 0.001$ & $0.216 \pm 0.001$ \\
$t_{\text {ast }}($ days $)$ & $0.217 \pm 0.001$ & $0.218 \pm 0.001$ & & \\
\hline
\end{tabular}

Note. Mean values from the MCMC are shown in parentheses. All other values are from the best-fit model. $\pi_{\mathrm{E}} \equiv \sqrt{\pi_{\mathrm{E}, N}^{2}+\pi_{\mathrm{E}, E}^{2}}, \phi_{\pi} \equiv \tan { }^{-1}\left(\pi_{\mathrm{E}, E} / \pi_{\mathrm{E}, N}\right)$, and $t_{\mathrm{ast}} \equiv \rho t_{\mathrm{E}}$ are derived quantities and are not fitted independently. All fluxes are on an 18th mag scale (e.g., $I_{s}=18-2.5 \log \left(f_{s}\right)$ ).

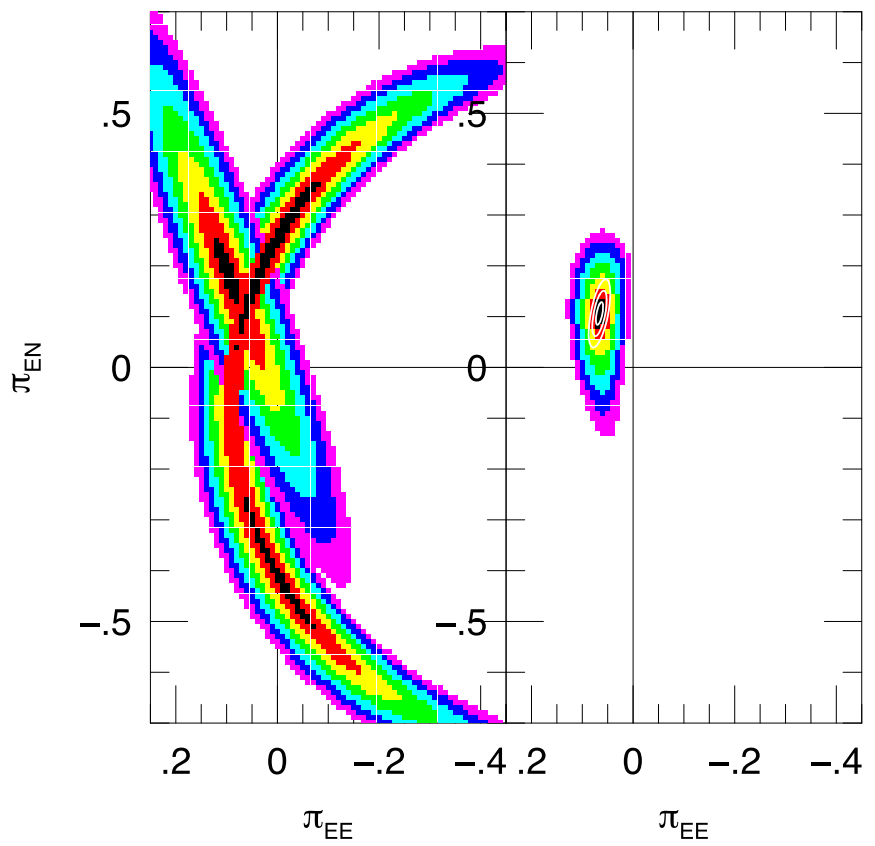

Figure 2. OGLE-2017-BLG-0406 parallax contours for the W+ solution. Left: the ground-only (elliptical) contours are derived from the covariance matrix from the MCMC, while the Spitzer-"only" (arc-like) contours are derived from the analytical expression in Equation (7). The colors (black, red, yellow, green, cyan, blue, magenta) represent $\Delta \chi^{2}<(1,4,9,16,25,36,49)$. Note that the $1 \sigma$ contours for the ground-only and Spitzer-"only" measurements overlap. Right: Colored contours are the $\Delta \chi^{2}$ values for the sum of the two $\chi^{2}$ distributions that are shown to the left. Despite the fact the each set of contours on the left provides essentially $1 \mathrm{D}$ information, the combination is well constrained in both dimensions. The white ellipses represent the $1 \sigma, 2 \sigma$, and $3 \sigma$ contours from the combined numerical fit to all of the data. The semi-analytic approach (colored contours) provides a very good, although not perfect, representation of the full numerical result. This shows that the semi-analytic approach enables one to accurately trace the information flow.

constraint happened to pass close to the origin. We will return to the role of this 1D parallax constraint after including Spitzer data into the analysis.

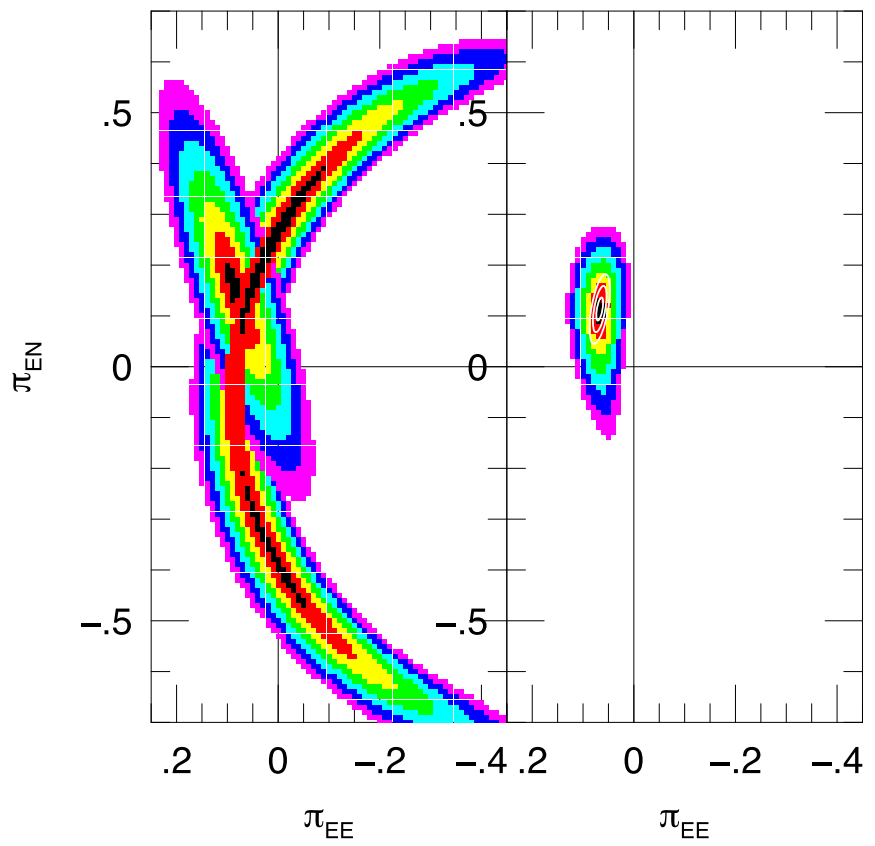

Figure 3. OGLE-2017-BLG-0406 parallax contours for the W- solution. Similar to Figure 2.

\section{Spitzer Parallax Analysis}

\subsection{Spitzer- "only" Parallax}

In principle, we could now proceed to incorporate the Spitzer data into a joint fit together with the ground-based data. We will do so in Section 5.2. First, however, it is important to examine how the Spitzer data and the ground-based data contribute information to the parallax measurement. The principal reason for doing so is that both data sets can be subject to systematic errors, which are of very different types and can affect the parallax measurement very differently. An important check for such systematics is whether the parallax information derived from each data set is consistent with the 
other. Failure of this test would provide clear evidence for systematics in one or both data sets. In addition, we will ultimately be making somewhat separate use of the magnitude and direction of the parallax vector. In order to understand how secure each of these components is, we will need to trace their origins in different combinations of Spitzer-based and groundbased information.

In Section 4, we showed that the ground-based data yielded essentially one-dimensional parallax information, with $\pi_{\mathrm{E}, \|}$ (the component parallel to the instantaneous projected direction of the Sun at $t_{0}$ ) measured about nine (for $\mathrm{W}+$ ) or five (for $\mathrm{W}-$ ) times more precisely than the orthogonal component $\pi_{\mathrm{E}, \perp}$. This is because the information for the latter comes from further out in the wings of the light curve (Smith et al. 2003; Gould 2004), which, for events like OGLE-2017-BLG-0406 that are not extremely long, is generally quite faint. This also means that the $\pi_{\mathrm{E}, \perp}$ component is much more sensitive to long-term trends in the data. In the present case, for which $\pi_{\mathrm{E}, \|} \simeq 0$, this means that the direction of the ground-based parallax vector is determined much more confidently than its magnitude.

The Spitzer light curves can also be affected by long-term trends in the data, which can affect the parallax measurement. In their analysis of 50 Spitzer events from 2015, Zhu et al. (2017) identified five with obvious trends in the data, and Koshimoto \& Bennett (2019) identified 14 more. There is only one case for which the causes of such trends have been investigated: KMT-2018-BLG-0029 (Gould et al. 2020). In that case, bright nearby blends with poorly determined positions were found to be likely to have generated trends, as the Spitzer camera rotated during the season. Because the source was faint and not well magnified, the trends were about $30 \%$ of the total observed flux variations. Nevertheless, after the trends were removed, the amplitude of the parallax measurement only changed by $20 \%$ (about $2 \sigma$ ). Thus, it is important to both carefully evaluate and minimize the impact of potential Spitzer systematics.

Refsdal (1966) originally analyzed satellite parallaxes prior to the time (Gould 1992) that it was recognized that the groundbased light curve alone would have any parallax information. Hence, although not explicitly stated, his was in essence a satellite-"only" analysis. The ground-based parameters $\left(t_{0, \oplus}\right.$, $\left.u_{0, \oplus}, t_{\mathrm{E}, \oplus}\right)$ were directly compared to the satellite parameters $\left(t_{0, \mathrm{sat}}, u_{0, \mathrm{sat}}, t_{\mathrm{E}, \mathrm{sat}}\right)$ to produce the parallax measurement

$$
\begin{aligned}
\pi_{\mathrm{E}} & =\frac{\mathrm{au}}{D_{\perp}}\left(\Delta \tau, \Delta u_{0}\right) ; \quad \Delta \tau \equiv \frac{t_{0, \mathrm{sat}}-t_{0, \oplus}}{t_{\mathrm{E}}} ; \\
\Delta u_{0} & \equiv u_{0, \mathrm{sat}}-u_{0, \oplus},
\end{aligned}
$$

where $\boldsymbol{D}_{\perp}$ is the projected separation of the satellite from Earth and where it was implicitly assumed that the Einstein timescales were the same $t_{\mathrm{E}}=t_{\mathrm{E}, \oplus}=t_{\mathrm{E}, \mathrm{sat}}$. The implicit idea (as illustrated in Figure 1 of Gould 1994 and first realized in Figure 1 of Yee et al. $2015 \mathrm{~b})$ is that $\left(t_{0, \mathrm{sat}}, u_{0, \mathrm{sat}}, t_{\mathrm{E}, \mathrm{sat}}\right)$ would be "observables" from the satellite, just as the corresponding quantities were from Earth. Note that Equation (4) has a fourfold degeneracy because $u_{0}$ is a signed quantity but only $\left|u_{0}\right|$ is determined directly from the light curve. See Figure 4 of Gould (2004) for the sign convention.

However, in real Spitzer microlensing events, the peak is very often not observed from space, primarily because there is a 3-10 day time delay between identifying the event and initiating satellite observations (Figure 1 from Udalski et al. 2015b). Hence, while Equation (4) remains formally valid, it may no longer express the parallax measurement in terms of "observables," because $\left(t_{0}, u_{0}\right)_{\text {sat }}$ may not be separately determined.

Gould (2019) generalized Refsdal's satellite-“only” analysis to the case of satellite data streams that did not cover the peak. He showed that if the source flux in the satellite observations was known and the baseline flux was measured, then each satellite measurement at finite magnification yields an exactly circular degeneracy in the $\pi_{\mathrm{E}}$ plane. In the presence of measurement errors, these circles become finite annuli. If these measurements cover the peak, then the corresponding circles intersect in exactly two places, which then reproduces the Refsdal (1966) fourfold degeneracy (two pairs, one each for $\left.\pm u_{0, \oplus}\right)$. See Figure 3 of Gould (2019). On the other hand, the osculating circles from a series of late-time measurements combine to form an extended arc. See Figure 1 of Gould (2019). Such arcs may yield exquisite 1D constraints on $\pi_{\mathrm{E}}$ while still providing almost no constraint on its amplitude $\pi_{\mathrm{E}}$. See the second row of Figure 2 from Zang et al. (2020) for an extreme example. Nevertheless, as that example makes clear, the addition of exterior information about the direction of $\pi_{\mathrm{E}}$ can then constrain $\pi_{\mathrm{E}}$ very well. The first case of such an arc appearing in a Spitzer-"only" analysis was OGLE-2018-BLG0596 (Jung et al. 2019). OGLE-2017-BLG-0406 has a qualitatively similar arc-like degeneracy.

We note that if $\left(t_{0}, u_{0}, t_{\mathrm{E}}\right)_{\oplus}$ are considered as known exactly, then for each trial value of $\pi_{\mathrm{E}}=\left(\pi_{\mathrm{E}, N}, \pi_{\mathrm{E}, E}\right)$, the remaining two parameters $\left(f_{s}, f_{b}\right)_{\text {Spitzer }}$ can be calculated analytically. That is, there are $\left(N_{\text {Spitzer }}+1\right)$ linear equations for two unknowns, where $N_{\text {Spitzer }}$ is the number of Spitzer measurements, $F\left(t_{k}\right)$. These are $N_{\text {Spitzer }}$ equations for the measurements,

$$
\begin{aligned}
y_{k} & =\sum_{i=1}^{2} a_{i} g_{i, k} \pm \sigma_{k} \quad y_{k} \equiv F\left(t_{k}\right), \\
a & \equiv\left(f_{s}, f_{b}\right)_{\text {Spitzer }} ; \quad g_{1, k} \equiv A\left(t_{k}\right), \quad g_{2, k} \equiv 1,
\end{aligned}
$$

plus one for the flux constraint,

$$
\begin{aligned}
y_{0} & =\sum_{i=1}^{2} a_{i} g_{i, 0} \pm \sigma_{0} \quad y_{0} \equiv f_{\mathrm{s}, \text { spitzer,constr }} \\
g_{1,0} & \equiv 1 \quad g_{2, k} \equiv 0
\end{aligned}
$$

Then one solves in the usual way,

$$
\begin{aligned}
& d_{i}=\sum_{\mu=0}^{N_{\text {Spizzer }}} \frac{y_{\mu} g_{i, \mu}}{\sigma_{\mu}^{2}} ; \quad b_{i, j}=\sum_{\mu=0}^{N_{\text {Spitzer }}} \frac{g_{i, \mu} g_{j, \mu}}{\sigma_{\mu}^{2}} ; \\
& c=b^{-1} \quad a_{i}=\sum_{j} c_{i, j} d_{j},
\end{aligned}
$$

with $c_{i, j}$ being the covariance matrix of the two parameters.

To evaluate the Spitzer-"only" parallax contours, we calculate $A_{\text {Spitzer }}\left(t_{k}\right)$ by fixing $\left(t_{0}, u_{0}, t_{\mathrm{E}}\right)_{\oplus}$ according to the $\mathrm{W}+$ and $\mathrm{W}-$ solutions shown in Table 3 and by fixing $\pi_{\mathrm{E}}=\left(\pi_{\mathrm{E}, N}, \pi_{\mathrm{E}, E}\right)$ at a grid of values. In Section 6 , we evaluate $f_{\text {s, spitzer,constr }}=11.10 \pm 0.15$. We find that the Spitzer errors must be renormalized by a factor 3.4 to achieve $\chi^{2} /$ dof $=1$. The arc in Figure 2 shows the resulting Spitzer-"only" contours for the $\mathrm{W}+$ solution. The diagonal contours represent the ground-only parallax measurement, which we have extended out to seven sigma analytically using the covariance matrix 
Table 4

Wide Models for Ground+Spitzer Data

\begin{tabular}{|c|c|c|}
\hline Parameters & Wide $(+,+)$ & Wide $(-,+)$ \\
\hline$\chi^{2} /$ dof & $29297.755 / 29310$ & $29295.132 / 29310$ \\
\hline$t_{0}\left(\mathrm{HJD}^{\prime}\right)$ & $7908.813 \pm 0.001$ & $7908.813 \pm 0.001$ \\
\hline$u_{0}\left(10^{-3}\right)$ & $9.281 \pm 0.028$ & $-9.281 \pm 0.028$ \\
\hline$t_{\mathrm{E}}$ (days) & $37.134 \pm 0.083$ & $37.133 \pm 0.085$ \\
\hline$s$ & $1.128 \pm 0.001$ & $1.128 \pm 0.001$ \\
\hline$q\left(10^{-4}\right)$ & $6.955 \pm 0.061$ & $6.970 \pm 0.090$ \\
\hline$\alpha(\mathrm{rad})$ & $0.993 \pm 0.001$ & $-0.993 \pm 0.001$ \\
\hline$\rho\left(10^{-3}\right)$ & $5.852 \pm 0.025$ & $5.843 \pm 0.025$ \\
\hline$\pi_{\mathrm{E}, N}$ & $0.126(0.111) \pm 0.021$ & $0.120(0.113) \pm 0.024$ \\
\hline$\pi_{\mathrm{E}, E}$ & $0.062(0.066) \pm 0.007$ & $0.065(0.067) \pm 0.007$ \\
\hline$\pi_{\mathrm{E}}$ & $0.140(0.130) \pm 0.016$ & $0.136(0.133) \pm 0.018$ \\
\hline$\phi_{\pi}$ & $0.455(0.549) \pm 0.127$ & $0.499(0.549) \pm 0.134$ \\
\hline$f_{S}(\mathrm{OGLE})$ & $1.459 \pm 0.004$ & $1.459 \pm 0.004$ \\
\hline$f_{B}(\mathrm{OGLE})$ & $0.106 \pm 0.004$ & $0.105 \pm 0.004$ \\
\hline$f_{S}($ Spitzer $)$ & $11.249 \pm 0.164$ & $11.210 \pm 0.180$ \\
\hline$f_{B}($ Spitzer $)$ & $-2.656 \pm 0.165$ & $-2.614 \pm 0.182$ \\
\hline$t_{\text {ast }}$ (days) & $0.217 \pm 0.001$ & $0.217 \pm 0.001$ \\
\hline
\end{tabular}

Note. Mean values from the MCMC are shown in parentheses. All other values are from the best-fit model. $\pi_{\mathrm{E}}, \phi_{\pi}$, and $t_{\mathrm{ast}}$ are derived quantities and are not fitted independently. All fluxes are on an 18th mag scale (e.g., $I_{s}=18-2.5 \log \left(f_{s}\right)$ ).

from the MCMC. Figure 3 shows the corresponding structures for the W-solution.

The most important feature is that the $1 \sigma$ contours from the two parallax measurements overlap. Hence, there is no tension at all between the two determinations. Second, the direction of $\pi_{\mathrm{E}}$ is essentially determined by the ground-based measurement. That is, even if the arc were displaced to the East or West, it would intersect the ground contours at a very similar polar angle. The only exception would be if it intersected very close to the origin. Third, the best-fit ground-based value of $\pi_{\mathrm{E}, \perp}$ plays only a very small role in the point of overlap of these two sets of contours, which is shown in the right hand panel of the figure. That is, even if $\pi_{\mathrm{E}, \perp}$ were displaced two sigma toward higher values, the overlap would occur in the same place. This means that the aspect of the ground-based data that is most vulnerable to systematic errors does not play much of a role in the final solution. The panel at the right shows that despite the fact that the ground-only and Spitzer-"only" measurements are effectively $1 \mathrm{D}$, they combine to form tight $2 \mathrm{D}$ constraints.

\subsection{Combined Spitzer and Ground-based Analysis}

We thus proceed to directly analyze the ground-based and Spitzer data jointly. The resulting microlensing-parameter estimates are given in Table 4, where in particular, we show two different representations of the parallax vector $\pi_{\mathrm{E}}$ - that is, in Cartesian $\left(\pi_{\mathrm{E}, N}, \pi_{\mathrm{E}, E}\right)$ and polar $\left(\pi_{\mathrm{E}}, \pi_{\phi}\right)$ coordinates. We evaluate the $\pi_{\mathrm{E}}$ covariance matrix and use this to generate $1 \sigma$, $2 \sigma$, and $3 \sigma$ contours, which are shown in the right panels Figures 2 and 3 as white ellipses. These show that the semianalytic approach described in Section 5.1 and displayed in these figures works quite well, although not perfectly. This good agreement confirms that there is strong physical basis for the arguments given in that section.

\section{Color-Magnitude Diagram}

We can derive the angular Einstein radius, $\theta_{\mathrm{E}}=\theta_{\star} / \rho$, because the finite-source size, $\rho$, is constrained from the lightcurve fitting, and the angular size of the source star, $\theta_{\star}$, can be derived from the extinction-corrected source color and brightness. The measurement of $\theta_{\mathrm{E}}$ gives the following massdistance relation of lens system,

$$
M=\frac{c^{2}}{4 G} \theta_{\mathrm{E}}^{2} \frac{D_{\mathrm{S}} D_{\mathrm{L}}}{D_{\mathrm{S}}-D_{\mathrm{L}}}=\frac{\theta_{\mathrm{E}}^{2}}{\kappa \pi_{\mathrm{rel}}} .
$$

\subsection{Calibration}

We derive the source magnitudes in the $V$ and $I$ bands by converting the instrumental source magnitude in MOA-Red and MOA- $V$ bands into the standard Kron-Cousin $I$-band and Johnson $V$-band scales using the following relations:

$$
\begin{aligned}
I_{\mathrm{OGLE}-\mathrm{III}}-R_{\mathrm{MOA}}= & (28.132 \pm 0.002) \\
& -(0.206 \pm 0.001)(V-R)_{\mathrm{MOA}}, \\
V_{\mathrm{OGLE}-\mathrm{III}}-V_{\mathrm{MOA}}= & (28.302 \pm 0.002) \\
& -(0.108 \pm 0.001)(V-R)_{\mathrm{MOA}} .
\end{aligned}
$$

From the light-curve fitting using these formulae, we obtain the source color and magnitude $(V-I)_{\mathrm{S}}=2.581 \pm 0.016$ and $I_{\mathrm{S}}=$ $17.603 \pm 0.011$. We also calibrate the CTIO $H$-band magnitude to 2MASS Carpenter (2001) scale with the following relation,

$$
H_{2 \text { mass }}=H_{\text {CTIO }}-3.917 \pm 0.009 \text {, }
$$

based on the stars within $120^{\prime \prime}$ of the target. We find the source magnitude $H_{\mathrm{S}}=14.696 \pm 0.010$ and derive color $(V-H)_{\mathrm{S}}=$ $5.488 \pm 0.016$ and $(I-H)_{\mathrm{S}}=2.907 \pm 0.015$.

\subsection{Source Angular Radius}

To obtain the intrinsic source color and magnitude, we use the red clump giants (RCG) centroid in the color-magnitude diagram (CMD) as a standard candle. Figures 4-6 show CMDs of stars within $120^{\prime \prime}$ of the target. The $V$ and $I$ magnitudes are from OGLE-III catalog, and the $H$ magnitude is from the VVV catalog, which is calibrated to the 2MASS scale, respectively. We find that the centroids of the RCGs in this field, which are indicated as filled red circles, are at $I_{\mathrm{RCG}}=16.302 \pm 0.045$, $(V-I)_{\mathrm{RCG}}=2.623 \pm 0.012,(V-H)_{\mathrm{RCG}}=5.517 \pm 0.030$, and $(I-H)_{\mathrm{RCG}}=2.886 \pm 0.016$ from these CMDs. From Nataf et al. (2016) and Bensy et al. (2013), we also find that the intrinsic magnitude and color of RCG should be $I_{\mathrm{RCG}, 0}=14.426 \pm 0.040,(V-I)_{\mathrm{RCG}, 0}=1.060 \pm 0.060$, $(V-H)_{\mathrm{RCG}, 0}=2.360 \pm 0.090$, and $(I-H)_{\mathrm{RCG}, 0}=1.300 \pm$ 0.060 . The color and magnitude of source and the centroid of RCG are summarized in Table 5. By subtracting the intrinsic RGC color and magnitude from the measured RGC positions in our CMDs, we find an extinction value of $A_{I, \mathrm{obs}}=1.876 \pm$ 0.060 and color excess values of $E(V-I)_{\mathrm{obs}}=1.563 \pm$ $0.061, E(V-H)_{\mathrm{obs}}=3.157 \pm 0.095$, and $E(I-H)_{\mathrm{obs}}=$ $1.586 \pm 0.062$.

The extinction can be determined most accurately if three colors are used (Bennett et al. 2010). Following Bennett et al. (2010) and Koshimoto et al. (2017), we fit them with the extinction law of Cardelli et al. (1989) and Nishiyama et al. (2008, 2009). Table 6 shows the results of fitting extinction values to those of extinction laws. We adopt the 


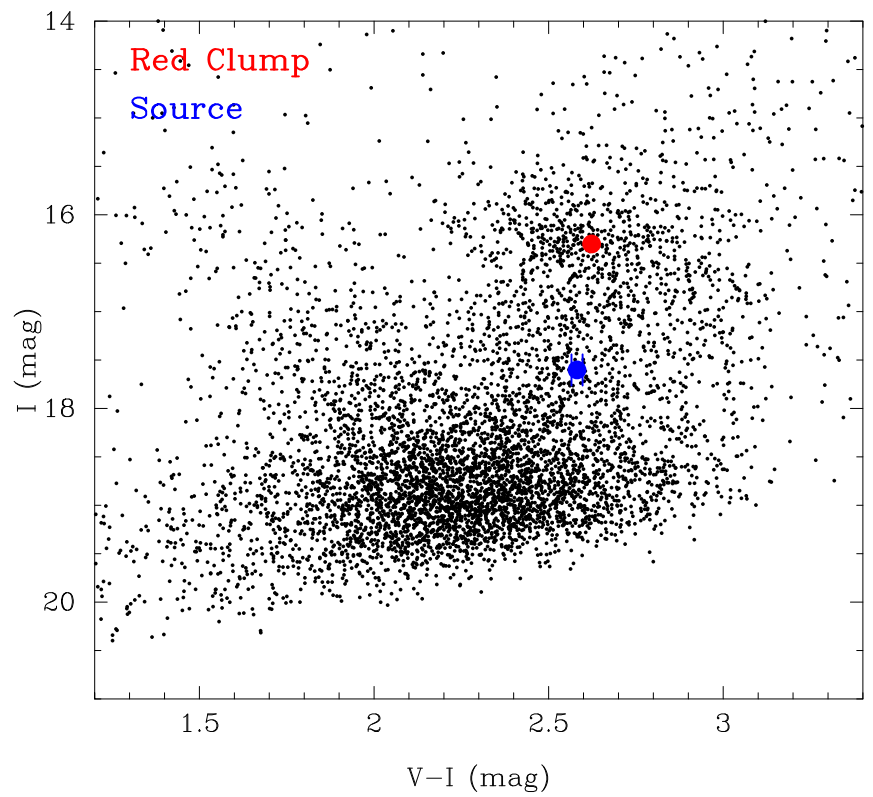

Figure 4. The $(V-I, I)$ color-magnitude diagram (CMD) of the OGLE stars within $120^{\prime \prime}$ of OGLE-2017-BLG-0406. The red-filled circle indicates the red clump giant (RCG) centroid, and the blue-filled circle indicates the source color and magnitude, respectively.

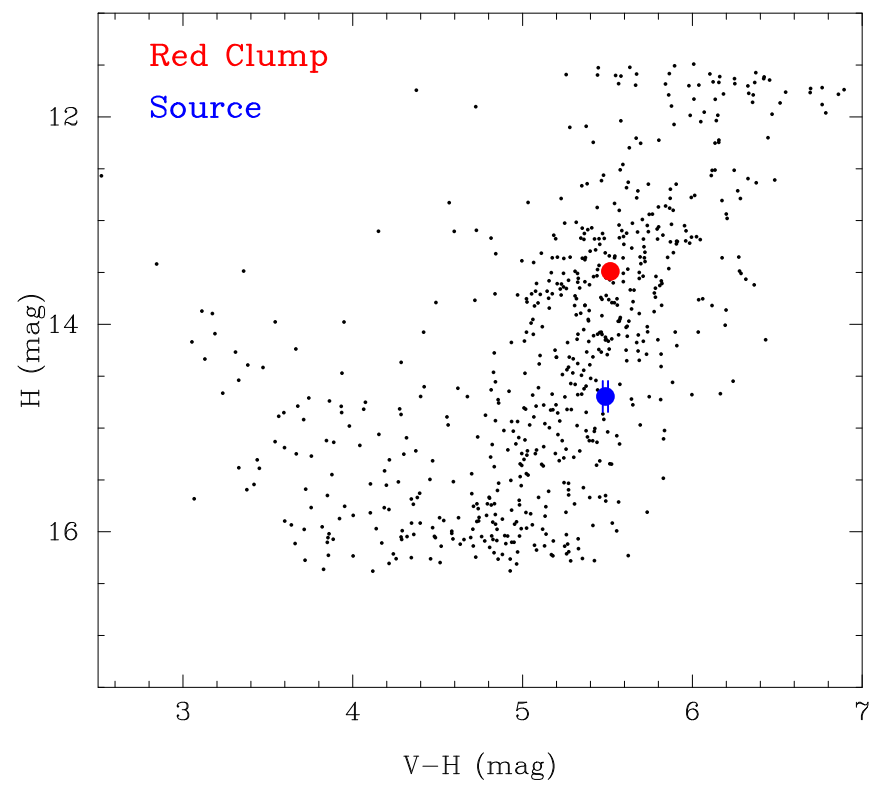

Figure 5. The $(V-H, H)$ color-magnitude diagram (CMD) of OGLE-2017BLG-0406. $V$ - and $H$-band magnitudes are calibrated to the Johnson $V$ and 2MASS scale, respectively. The red-filled circle indicates the red clump giant (RCG) centroid, and the blue-filled circle indicates the source color and magnitude, respectively.

$R_{J K V I} \equiv E(J-K s) / E(V-I)=0.3347$ value from Nataf et al. (2013) for the event coordinates. We see that the $\chi^{2}$ value using Nishiyama et al. (2008) extinction law is the smallest, and the extinction values agree with our measurement from our CMDs. Thus, we decide to use the results from Nishiyama et al. (2008) extinction law for the rest of the analysis.

The extinction-corrected magnitude and color of the source indicate that it sits about 1.27 mag below the red clump centroid on the giant branch. A comparison to isochrones

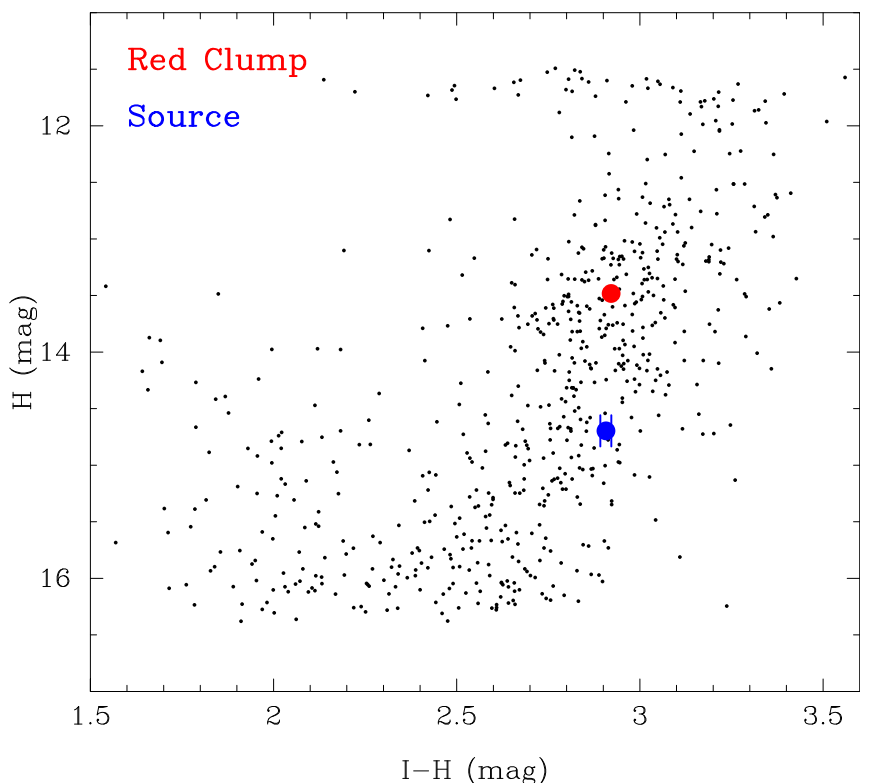

Figure 6. The $(I-H, H)$ color-magnitude diagram (CMD) of OGLE-2017BLG-0406. $I$ - and $H$-band magnitudes are calibrated to the Cousins $I$ and 2MASS scale, respectively. The red-filled circle indicates the red clump giant (RCG) centroid, and the blue-filled circle indicates the source color and magnitude, respectively.

following Bennett et al. (2018a, 2018b) indicates that that source star is located on the giant branch in the Galactic bulge. Stars of similar color and magnitude that reside in the foreground or background have a negligible probability to be lensed because of an extremely low number density. So, we conclude the the source star almost certainly resides in the Galactic bulge.

Because the most precise determination comes from the $(V-H)$ and $H$ relation (Bennett et al. 2015), we use the following relation to estimate $\theta_{\star}$,

$$
\log \theta_{\mathrm{LD}}=0.536654+0.072703(V-H)_{\mathrm{S}, 0}-0.2 H_{\mathrm{S}, 0},
$$

where $\theta_{\mathrm{LD}}$ is the limb-darkened stellar angular diameter (Boyajian et al. 2014). This relation comes from a private communication with Boyajian by Bennett et al. (2015). For the best-fit parameter, we get $\theta_{\star}=\theta_{\mathrm{LD}} / 2=3.472 \pm 0.085 \mu \mathrm{as}$.

\subsection{Source Angular Radius Using IRSF Data}

We also derive $\theta_{\star}$ using relation between $\left(V-K_{\mathrm{S}}\right)_{\mathrm{S}, 0}$ and $K_{\mathrm{S}, \mathrm{S}, 0}$ obtained from IRSF data. From the light-curve fitting, we get $\left(V-K_{\mathrm{S}}, K_{\mathrm{S}}\right)_{\mathrm{S}}=(5.262,14.923) \pm(0.091,0.093)$. From the results of Nishiyama et al. (2008), we also get the extinction in $K_{\mathrm{S}}$-band $A_{\mathrm{K}_{\mathrm{S}}}=0.222 \pm 0.005$ and color excess $E\left(V-K_{\mathrm{S}}\right)=3.250 \pm 0.077$. Thus we find $\left(V-K_{\mathrm{S}}, K_{\mathrm{S}}\right)_{\mathrm{S}, 0}=$ $(2.011,14.701) \pm(0.122,0.090)$. To estimate $\theta_{\star}$, we use the following equation from Kervella et al. (2004):

$$
\log \theta_{\mathrm{LD}}=0.5170+0.0755(V-K)_{\mathrm{S}, 0}-0.2 K_{\mathrm{S}, 0} .
$$

This gives $\theta_{\star}=2.68 \pm 0.14 \mu \mathrm{as}$, which is inconsistent with the one from $(V-H, H)_{S, 0}$. We also get $H_{\mathrm{S}}=15.173 \pm$ 0.090 from light-curve fitting of IRSF data, which is about 0.5 mag fainter than the one we get from CTIO data. This is likely because we only have three observations from IRSF for 
Table 5

The Source Color and Magnitude

\begin{tabular}{|c|c|c|c|c|}
\hline & $I$ & $V-I$ & $\bar{V} V-H$ & $\overline{I I-H}$ \\
\hline RCG (measured from CMDs) & $16.302 \pm 0.045$ & $2.623 \pm 0.012$ & $5.517 \pm 0.030$ & $2.886 \pm 0.016$ \\
\hline RCG (extinction-corrected) & $14.426 \pm 0.040$ & $1.060 \pm 0.060$ & $2.360 \pm 0.090$ & $1.300 \pm 0.060$ \\
\hline Source (measured from light-curve fitting) & $17.603 \pm 0.011$ & $2.581 \pm 0.016$ & $5.488 \pm 0.016$ & $2.907 \pm 0.015$ \\
\hline
\end{tabular}

Note.

${ }^{a}$ Extinction-corrected magnitudes using the Nishiyama et al. (2008) extinction law from Table 6.

Table 6

Comparison of the Extinction Based on Different Extinction Laws

\begin{tabular}{lcccc}
\hline \hline Extinction law & None & Cardelli et al. (1989) & Nishiyama et al. (2009) & Nishiyama et al. (2008) \\
\hline$A_{V}$ & $3.437 \pm 0.086$ & $3.565 \pm 0.055$ & $3.497 \pm 0.062$ & $\mathbf{3 . 4 7 2} \pm \mathbf{0 . 0 8 2}$ \\
$A_{I}$ & $1.876 \pm 0.060$ & $1.982 \pm 0.050$ & $1.931 \pm 0.050$ & $\mathbf{1 . 9 1 1} \pm \mathbf{0 . 0 6 6}$ \\
$A_{H}$ & $0.364 \pm 0.103$ & $0.583 \pm 0.018$ & $0.467 \pm 0.012$ & $\mathbf{0 . 3 5 8} \pm \mathbf{0 . 0 0 9}$ \\
$E(V-I)$ & $1.563 \pm 0.061$ & $1.587 \pm 0.037$ & $3.031 \pm 0.038$ & $\mathbf{1 . 5 6 1} \pm \mathbf{0 . 0 5 3}$ \\
$E(V-H)$ & $3.157 \pm 0.095$ & $2.987 \pm 0.047$ & $1.464 \pm 0.052$ & $\mathbf{3 . 1 1 5} \pm \mathbf{0 . 0 7 4}$ \\
$E(I-H)$ & $1.586 \pm 0.062$ & $1.401 \pm 0.033$ & $2.70 / 1$ & $\mathbf{1 . 5 5 4} \pm \mathbf{0 . 0 6 1}$ \\
$\chi^{2} /$ dof & $\cdots$ & $11.60 / 1$ & $2.66 / 2$ \\
\hline
\end{tabular}

Note. The bold values are the values used for the analysis.

this event, and our normal procedure for renormalizing error bars is not very reliable. Therefore, we adopt a $\theta_{\star}$ value derived from the CTIO $V-H$ relations for the rest of the analysis.

\subsection{Color-color Relation for Spitzer}

We construct an $I H L$ color-color diagram by matching field stars from OGLE-IV, VVV, and our own Spitzer photometry. We restrict attention to stars in the neighborhood of the clump, $(2.75<(I-H)<3.10) \times(16<I<17.6)$, and show the cross matches is Figure 7 . We fit these points to a straight line and find $(I-L)=1.289[(I-H)-2.90]+2.215 \pm 0.008$. We find from regression $\left(I-H_{\mathrm{CT13}}\right)_{s}=1.109 \pm 0.004$, and so $\left(I-H_{\mathrm{VVV}}\right)_{s}=2.898 \pm 0.010$. Hence this error in $(I-H)_{s}$ propagates to an error of $0.013 \mathrm{mag}$ in $(I-L)_{s}$. To this, we must add in quadrature the error in the relation at the color of the source (0.01 mag), yielding finally $(I-L)=2.215 \pm 0.015$.

This approach implicitly assumes that the $\sim 0.1$ mag scatter seen in Figure 7 is overwhelmingly due to measurement error rather than intrinsic variation. This is justified by the Bessell \& Brett (1988) study of color-color relations based on bright isolated stars with excellent photometry, which found very small scatter.

\section{Location and Proper Motion of the Source}

The physical parameters that can be derived from the microlensing solution alone appear to be quite typical of Galactic microlensing events. That is, from $\theta_{\mathrm{E}}=0.59$ mas and $\pi_{\mathrm{E}}=0.13$, we can derive $M=\theta_{\mathrm{E}} / \kappa \pi_{\mathrm{E}}=0.56 M_{\odot}$ and $\pi_{\text {rel }}=0.077$ mas, which would be consistent with a disk lens at $D_{\mathrm{L}} \sim 5 \mathrm{kpc}$ and a bulge source located at $D_{\mathrm{S}} \sim 8 \mathrm{kpc}$, as we have inferred from the source brightness and color. This would also be consistent with the direction of lens-source relative motion $\phi_{\pi} \equiv \tan ^{-1}\left(\pi_{\mathrm{E}, E} / \pi_{\mathrm{E}, N}\right)=$ $32^{\circ} \pm 8^{\circ}$ (and the observed amplitude of this motion

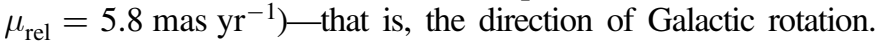

This is the direction that would be expected for a typical bulge source and a typical disk lens.

\subsection{Gaia Proper Motion of the Source}

However, this seemingly clear picture appears to be contradicted by the Gaia source proper motion

$$
\boldsymbol{\mu}_{s}(N, E)=(0.105,0.124) \pm(0.752,0.840) \quad \text { (Gaia) }
$$

that is, moving synchronously with the flat disk-rotation curve, rather than the mean motion of the bulge.

The Gaia measurement is very difficult to understand within the context of the microlensing solution. It would imply that the lens is moving relative to the source at $v_{\text {rel }} \simeq \mu_{\text {rel }} D_{\mathrm{L}}=$ $135 \mathrm{~km} \mathrm{~s}^{-1}\left(D_{\mathrm{L}} / 5 \mathrm{kpc}\right)$ in the prograde direction. While not impossible, this would be a very rare star. Another alternative to consider is that $\pi_{\text {rel }}$ is actually somewhat smaller (due to measurement errors of $\theta_{\mathrm{E}}$ and $\pi_{\mathrm{E}}$ ) so that the lens could be in the bulge. However, the implied motion of the lens (12 mas $\mathrm{yr}^{-1}$ relative to the mean motion of the bulge) would be extremely rare $\mathcal{O}\left(10^{-4}\right)$ for a bulge star. Thus, the Gaia propermotion measurement of the source would imply that the otherwise quite expected microlensing parameters were either incorrect or had extremely unusual implications.

This is one of two lines of argument that led us to suspect that the Gaia measurement was actually incorrect. The second was that in the course of constructing a cleaned Gaia propermotion diagram of neighboring clump stars, we noticed that stars with parallax/error ratios $\pi / \sigma(\pi)<-2$ were preferentially extreme proper-motion outliers. That is, although the proper motions of the majority of such stars were distributed similarly to those of stars with more typical parallaxes, about $10 \%$ had proper-motion vectors near the edges or even outside the normal distribution and so were most likely to be the result of catastrophic errors. The Gaia parallax for the source star is 


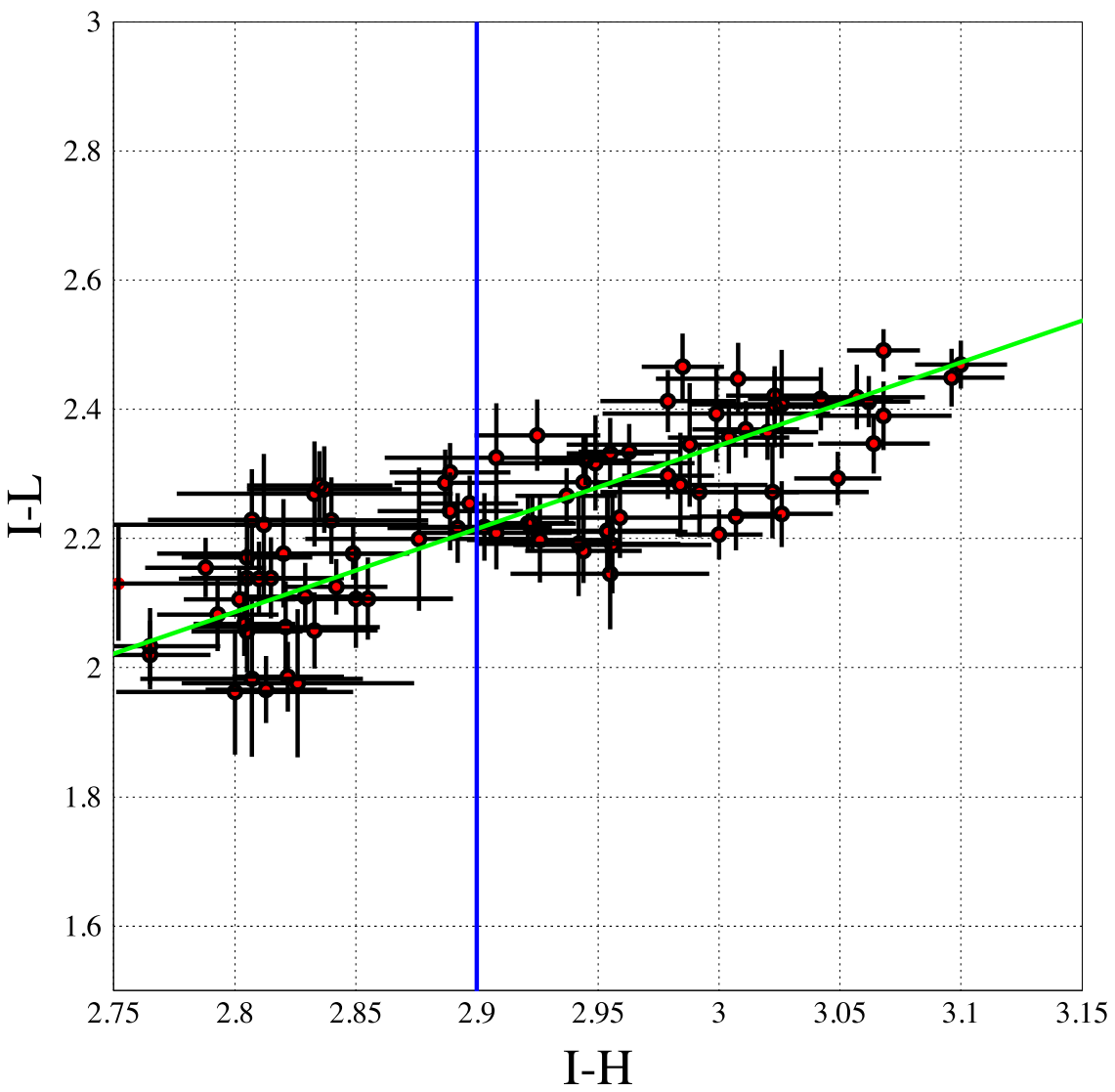

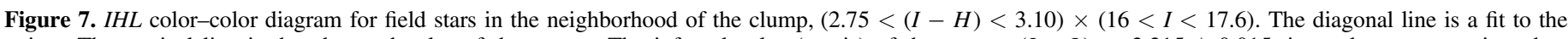

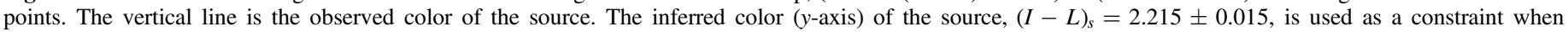
incorporating the Spitzer data into the fit.

$\pi=-0.96 \pm 0.42$ mas. Thus, based on our small statistical study, and even without any external reason to suspect the measurement, the strong negative parallax implied a $\sim 10 \%$ probability of a catastrophic error. We also note that this star has an "astrometric excess noise sig" of 3.19. However, we show below that this is actually substantially below the median of a well-behaved "clean clump and near-clump sample." So this value is not, in itself, a reason to be suspicious of this star.

\subsection{OGLE Proper Motion of the Source}

There is a long history of OGLE proper measurements of bulge sources dating back to the Sumi et al. (2004) catalog based on OGLE-II data. While there are no published catalogs based on the subsequent OGLE surveys, individual proper-motion measurements based on OGLE-IV are potentially more precise by a factor of several tens due to a five-times longer baseline and equal or higher cadence (Skowron et al. 2016b; Mróz et al. 2018; Chung et al. 2019; Shvartzvald et al. 2019). We apply this same technique to the OGLE-2017-BLG-0406 source and find, in the OGLE-IV reference frame tied to 1050 red clump stars within a $(6.5 \times 6.5)$ square, $\boldsymbol{\mu}_{s, \text { OGLE-IV }}(N, E)=(0.923,-3.147) \pm$ $(0.163,0.182)$ mas $\mathrm{yr}^{-1}$, where the errors are derived by assuming that errors of the individual position measurements are equal to the rms scatter about the best-fit straight line (i.e., $\sigma(N, E)=(10,11)$ mas $)$, which corresponds to about 0.04 OGLE pixels. Figure 8 shows the 324 data points during the period 2010-2019 that went into this measurement.

We then align the local OGLE-IV proper-motion frame to the Gaia frame by cross matching common stars. For this purpose, we consider Gaia stars within $\Delta \theta<3^{\prime}$ and with "astrometric excess noise sig" $<10$ and then further restrict to our "clean clump and near-clump sample," which is defined by $16<G<18,2<\left(B_{P}-R_{P}\right)<3, \sigma\left(\mu_{\text {R.A }}\right)<0.6$ mas yr $^{-1}$,

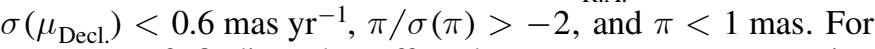
purposes of finding the offset between two proper-motion frames, there is no reason to restrict to clump stars. However, the clump (and near-clump) sample allows us to identify and reject several data classes that are prone to catastrophic errors. We find $\Delta \boldsymbol{\mu}(N, E)=\boldsymbol{\mu}_{\text {Gaia }}-\boldsymbol{\mu}_{\text {OGLE-IV }}=(-5.552,-3.391) \pm$ $(0.042,0.052)$ mas $^{-1} \mathrm{r}^{-1}$, based on an initial set of 394 stars from which we eliminate nine and six three-sigma outliers, respectively. Hence, we obtain $\boldsymbol{\mu}_{\mathrm{s}, \mathrm{Gaia}}=\boldsymbol{\mu}_{s, \mathrm{OGLE}-\mathrm{IV}}+\Delta \boldsymbol{\mu}=$ $(-4.63,-6.54) \pm(0.17,0.19)$.

While these small formal errors accurately reflect the OGLEIV measurement of the "catalog star" associated with the microlensed source, this catalog star is composed of both the source and a very small amount of blended light. Subtracting the precisely determined source flux from the somewhat more uncertain baseline flux of the catalog star, this blended flux is about $7 \%$ of the total. The true number could be slightly more or less. To take account of the possibly different proper motion of the blend, we augment the error in the source proper motion

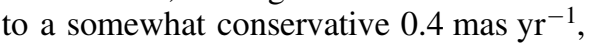

$$
\boldsymbol{\mu}_{s}(N, E)=(-4.63,-6.54) \pm(0.40,0.40) .
$$

(OGLE-IV).

Note that Equations (14) and (15) differ by about 8.1 mas $\mathrm{yr}^{-1}$ or about $10 \sigma$ using the reported Gaia uncertainties. 


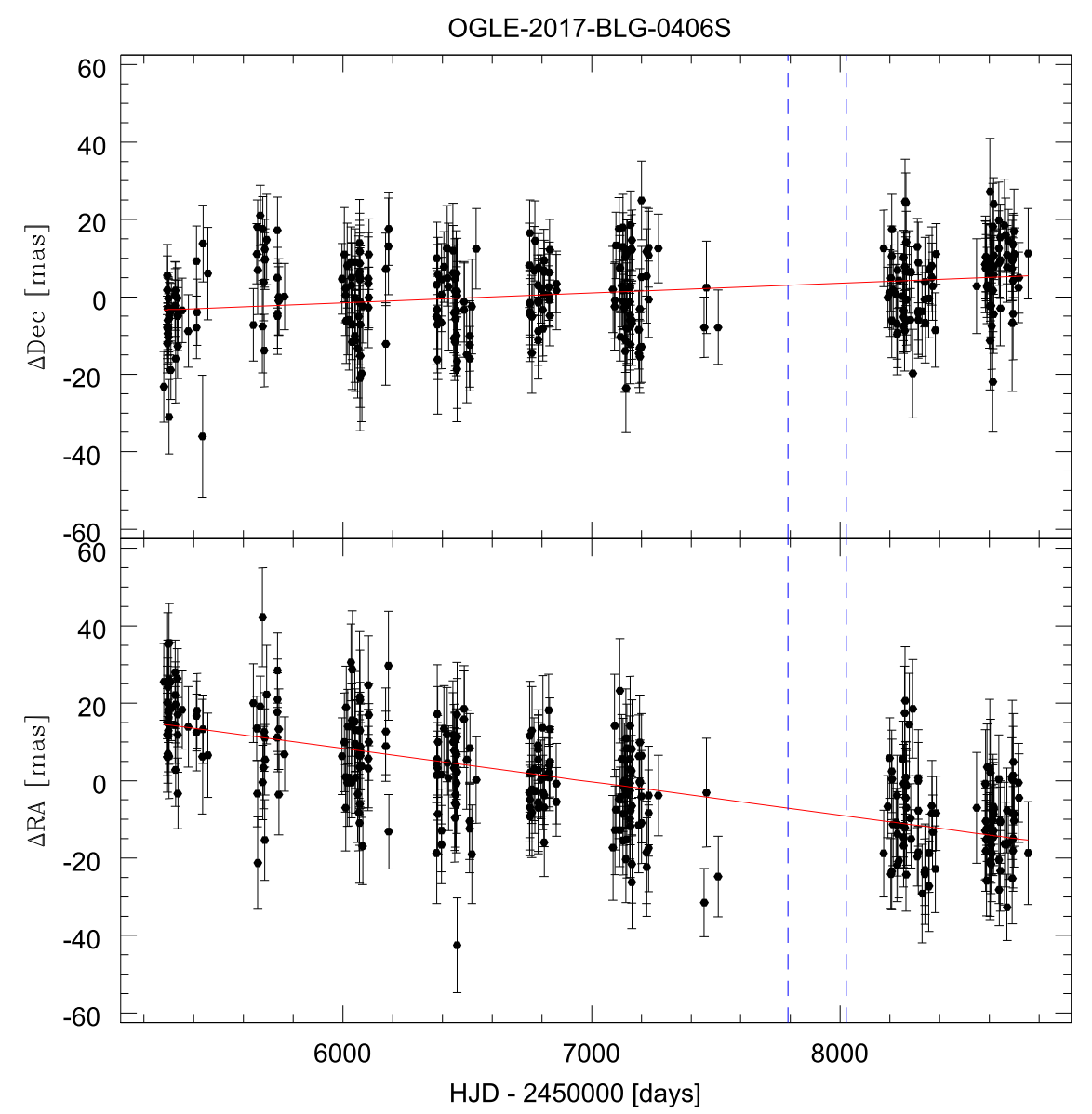

Figure 8. Individual position measurements converted to mas from OGLE-IV pixels ( 0 !! 26) on the $y$-axis (north, upper) and negative- $x$-axis (east, lower) of the detector. The observed slopes are $0.92 \pm 0.16$ mas yr$^{-1}$ (north) and $-3.15 \pm 0.18 \mathrm{mas} \mathrm{yr}^{-1}$ (east). By contrast, the measurements reported by Gaia would yield

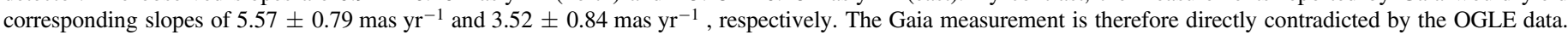

Our threshold of "astrometric excess noise sig" may appear at first site to be too generous. However, we find that in our final sample of 394 OGLE-Gaia matches, a fraction $(6,15,30,48) \%$ lie below $(1,2,3,4)$, respectively, with a median of 4.1. Yet, the sample as whole has well-behaved proper motions, with only $1 \%-2 \%$ three-sigma outliers relative to OGLE.

To find the offset $\Delta \boldsymbol{\mu}$ between the Gaia and OGLE-IV systems, we fit the proper-motion differences to a quadratic function of position centered at the lensed-source position. Because the formal Gaia errors were several times larger than the formal OGLE-IV errors, we considered only the former, and we rescaled these errors to enforce $\chi^{2} /$ dof $=1$. This yielded rescaling factors of 2.22 and 2.14 in the north and east directions, respectively. Figure 9 shows the proper-motion offsets as a function of each Equatorial coordinate. This figure shows some large-scale structure, which is removed by the quadratic fits, as well as some small-scale structure, which is not. However, this small-scale structure is relatively isolated and has a amplitude of a few tenths mas $\mathrm{yr}^{-1}$, so it is unlikely to account for the increased scatter, which is of order 1 mas $\mathrm{yr}^{-1}$. Rather, the most likely source of the majority of this additional scatter is underestimation of Gaia errors, which is exactly what is corrected by our errorrenormalization procedure. To further explore this idea, for each star in our "clean clump and near-clump sample" (but now re-including the stars with $\pi / \sigma(\pi)<-2$ ), we calculate a parallax offset parameter $\eta=\left(\pi-\pi_{0}\right) / \sigma(\pi)$, where $\pi_{0}=\pi_{\text {bulge }}-\pi_{\text {zpt }}=$ $70 \mu \mathrm{as}$, and we adopt $\pi_{\text {bulge }}=120 \mu$ as for the mean parallax of the clump and $\pi_{\mathrm{zpt}}=50 \mu$ as for zero-point offset of the Gaia parallax system. After restricting attention to $|\eta|<4$, we find $\langle\eta\rangle=-0.43$ (compared to zero expected), $\sigma(\eta)=1.31$ (compared to unity expected), and $\sqrt{\left\langle\eta^{2}\right\rangle}=1.38$ (compared to unity expected). If the error properties of the proper motions were similar to those of the parallaxes (as would be expected), then these numbers would partially explain the higher-than-expected scatter in the Gaia-OGLE-IV comparison.

One possible source of this Gaia astrometry error is blending in the crowded Galactic bulge field. Gaia has an asymmetric PSF that can lead to blending with another star at a separation of $\sim 0$ " 15 in some passes and not others. Such a circumstance would likely invalidate the Gaia astrometry, which could lead to negative parallaxes and proper-motion errors.

\section{Physical Parameters}

Because the microlens parallax vector, $\pi_{\mathrm{E}}$, the amplitude of the lens-source relative proper motion, $\mu_{\text {rel }}$, and the source proper motion, $\boldsymbol{\mu}_{s}$, are all well measured, and the source distance, $D_{\mathrm{S}}$, is constrained to reside in the Galactic bulge, we 

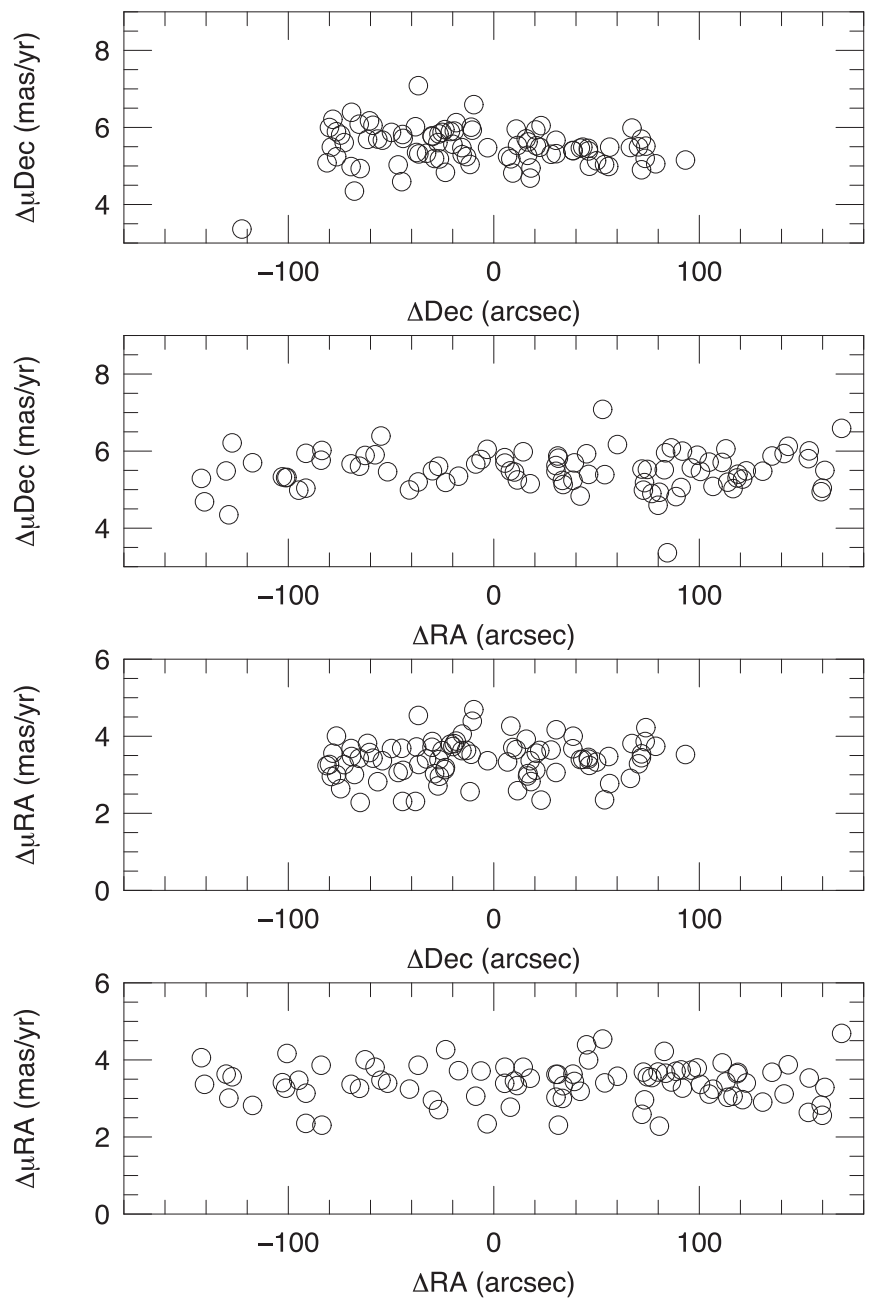

Figure 9. Offset of OGLE-IV proper motions relative to Gaia as a function of position in Equatorial coordinates (as indicated).

can directly calculate the lens physical parameters, namely ${ }^{62}$

$$
\begin{aligned}
M_{\mathrm{host}} & =\frac{\theta_{\mathrm{E}}}{(1+q) \kappa \pi_{\mathrm{E}}}, \quad \pi_{\mathrm{rel}}=\theta_{\mathrm{E}} \pi_{\mathrm{E}}, \\
\boldsymbol{\mu}_{\text {rel }} & =\frac{\boldsymbol{\pi}_{\mathrm{E}}}{\pi_{\mathrm{E}}} \mu_{\text {rel }}, \quad(\pi, \boldsymbol{\mu})_{\mathrm{L}}=(\pi, \boldsymbol{\mu})_{\mathrm{S}}+(\pi, \boldsymbol{\mu})_{\text {rel }} .
\end{aligned}
$$

We compute these quantities, as well as $M_{\text {planet }}, \boldsymbol{\mu}_{\text {rel, } \mathrm{H}}, a_{\perp}$, and $\boldsymbol{v}_{\mathrm{L}}$, from the MCMC, using a Galactic prior, and we report the results in Table 7 and Figures 10 and 11. The host star mass is denoted by $M_{\text {host }}$, and the planet mass is given by $M_{\text {planet }}=q M_{\text {host }}$.

We use a different Galactic prior than previous microlensing analyses (Sumi et al. 2011; Bennett et al. 2014; Zhu et al. 2017) because it is now clear that these older model have several incorrect features. One such feature is the varying Galactic-disk velocity dispersion as a function of the Galactocentric distance, $R$, which increases when $R$ gets smaller, while the disk scale height decreases with decreasing $R$. Another important feature is the changing distribution of the azimuthal velocity $V_{\phi}$ as a function of Galactocentric distance, $R$. If we define the circular velocity $V_{c}(R)$ as the velocity of a circular orbit at a distance $R$,

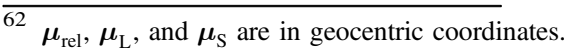

Table 7

Lens Physical Parameters

\begin{tabular}{lcc}
\hline \hline Parameter & Units & Values \\
\hline$M_{\text {host }}$ & $M_{\odot}$ & $0.56 \pm 0.07$ \\
$M_{\text {planet }}$ & $M_{\text {Jup }}$ & $0.41 \pm 0.05$ \\
$D_{\mathrm{S}}$ & $\mathrm{kpc}$ & $8.8 \pm 1.2$ \\
$D_{\mathrm{L}}$ & $\mathrm{kpc}$ & $5.2 \pm 0.5$ \\
$a_{\perp}$ & $\mathrm{au}$ & $3.5 \pm 0.3$ \\
$a_{3 \mathrm{~d}}$ & $\mathrm{au}$ & $4.1_{-0.7}^{+2.1}$ \\
$\theta_{\mathrm{E}}$ & $\mathrm{mas}$ & $0.593 \pm 0.012$ \\
$\mu_{\text {rel }}$ & $5.84 \pm 0.12$ \\
$\mu_{\text {rel,H,N }}$ & $\mathrm{mas} \mathrm{yr}^{-1}$ & $5.1 \pm 1.0$ \\
$\mu_{\text {rel,H,E }}$ & $\mathrm{mas} \mathrm{yr}^{-1}$ & $3.39 \pm 0.37$ \\
$v_{\mathrm{L}, l}$ & $\mathrm{mas} \mathrm{yr}^{-1}$ & $230 \pm 33$ \\
$v_{\mathrm{L}, b}$ & $\mathrm{~km} \mathrm{~s}^{-1}$ & $64 \pm 8$ \\
$V_{\mathrm{S}}$ & $\mathrm{km} \mathrm{s}^{-1}$ & $20.187 \pm 0.020$ \\
$I_{\mathrm{S}}$ & $\mathrm{mag}^{-1}$ & $17.606 \pm 0.020$ \\
$H_{\mathrm{S}}$ & $\mathrm{mag}$ & $14.697 \pm 0.020$ \\
$V_{\mathrm{L}}$ & $\mathrm{mag}$ & $26.1 \pm 0.9$ \\
$I_{\mathrm{L}}$ & $\mathrm{mag}$ & $22.7 \pm 0.7$ \\
$H_{\mathrm{L}}$ & $\mathrm{mag}$ & $19.6 \pm 0.5$ \\
\hline & $\mathrm{mag}$ & \\
\hline & & \\
\hline
\end{tabular}

then we find that there are more stars with $V_{\phi}<V_{c}$ than stars with $V_{\phi}>V_{c}$ at a given Galactocentric distance $R$, where $V_{c}$ is the circular velocity at $R$. Both of these features are observed in the Gaia DR2 data (Gaia Collaboration et al. 2018). N. Koshimoto et al. (2020 in preparation) developed a Galactic model that is based on the Shu distribution function model by Sharma et al. (2014), but modified so that the mean velocity and velocity dispersion as a function of the Galactocentric distance $R$ and the height from the Galactic plane $z$ match the Gaia DR2 data (Gaia Collaboration et al. 2018). Table 8 summarizes the distribution of Galactic transverse velocities in this Galactic model. It gives the median and 1 and $2 \sigma$ values for the stellar velocities for thin disk, thick disk, bulge, and all stars (i.e., the median and 15.85, 84.15, 2.28, and 97.72 percentiles of the transverse velocity distribution). The Galactic circular velocity in the Solar neighborhood is $V_{c}=238.8 \mathrm{~km} \mathrm{~s}^{-1}$ in our model. We used this model as the Galactic prior to calculate the lens properties.

We can determine the heliocentric proper motion of the lens, $\boldsymbol{\mu}_{\mathrm{L}, \mathrm{H}}$, because we have measured the microlensing parallax vector, $\pi_{\mathrm{E}}$, using

$$
\begin{aligned}
\boldsymbol{\mu}_{\mathrm{L}, \mathrm{H}} & \equiv \boldsymbol{\mu}_{\mathrm{L}}+\frac{\pi_{\mathrm{L}}}{\mathrm{au}} \boldsymbol{v}_{\oplus, \perp} ; \\
\boldsymbol{v}_{\oplus, \perp}(N, E) & =(+0.69,+28.27) \mathrm{km} \mathrm{s}^{-1},
\end{aligned}
$$

where $\boldsymbol{v}_{\oplus, \perp}$ is the projected velocity of Earth at $t_{o, \oplus}$. We can determine the velocity of the lens system with

$$
\begin{aligned}
& \boldsymbol{v}_{\mathrm{L}}=D_{\mathrm{L}} \boldsymbol{\mu}_{\mathrm{L}, \mathrm{H}}+\boldsymbol{v}_{\odot, \perp} \\
& \boldsymbol{v}_{\odot, \perp}(l, b)=(+250.8,+7) \mathrm{km} \mathrm{s}^{-1},
\end{aligned}
$$

and $v_{\odot}$ is the motion of the Sun in the Galactic frame, which includes its peculiar motion relative to the circular velocity, $V_{c}\left(R_{0}\right)$, at the solar circle, $R_{0}$, in a Galaxy-centered coordinate system. The lens velocity, $v_{\mathrm{L}}=\left(v_{\mathrm{L}, l}, v_{\mathrm{L}, b}\right)$, can then be compared with the median velocity $\left(\widetilde{v}_{l}, \widetilde{v}_{b}\right)$ of the Galactic stars at the distance of the lens system, $D_{\mathrm{L}}=5.2 \pm 0.5 \mathrm{kpc}$, as indicated in Table 8. 

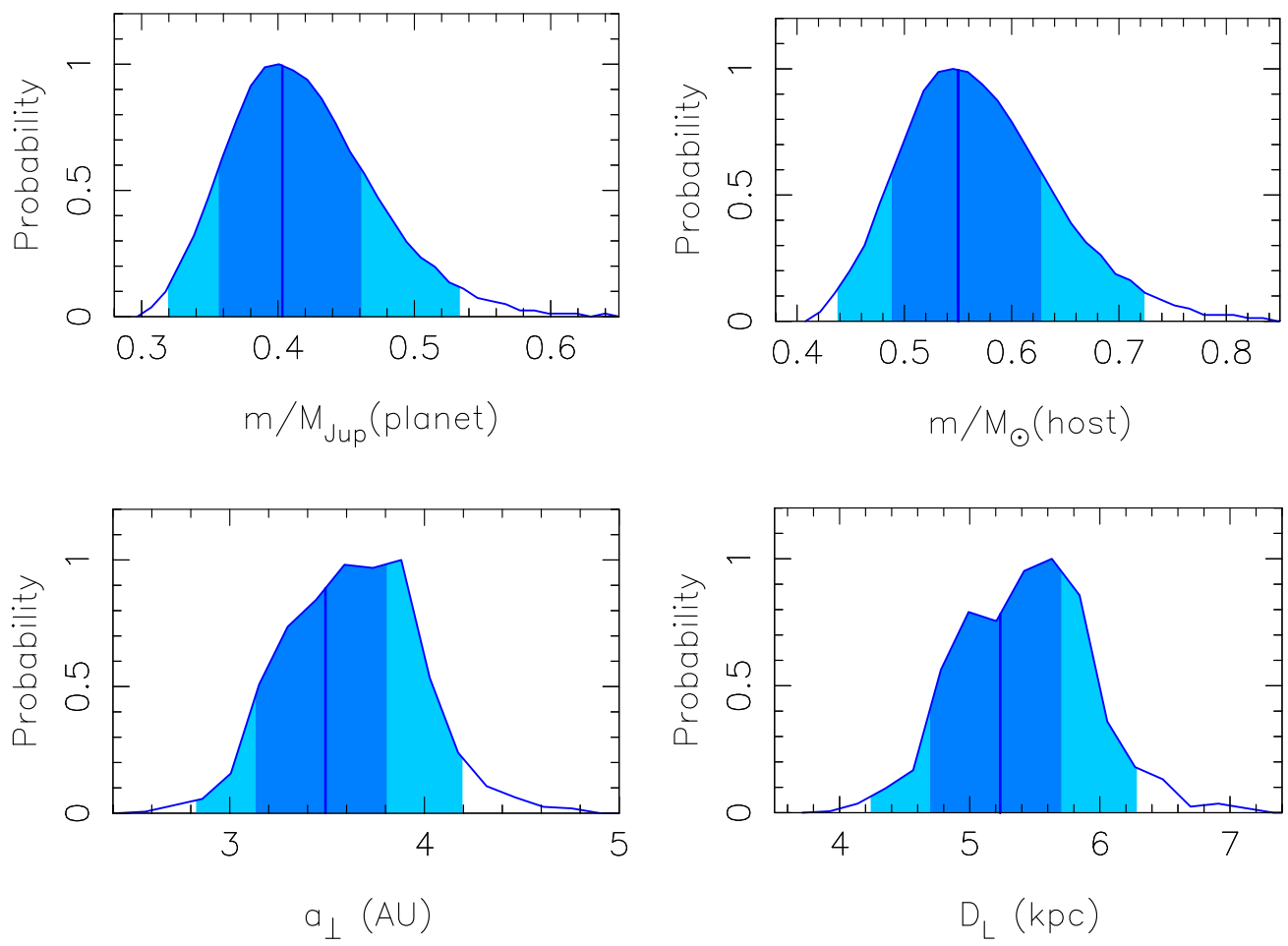

Figure 10. Probability distributions of lens properties of planetary mass, $M_{\text {planet }}$, host star mass, $M_{\text {host }}$, projected separation, $a_{\perp}$, and distance, $D_{\mathrm{L}}$, from our Bayesian analysis. The dark and light blue regions indicate the $68.3 \%$ and $95.4 \%$ confidence intervals, and the vertical blue lines indicate the median value.
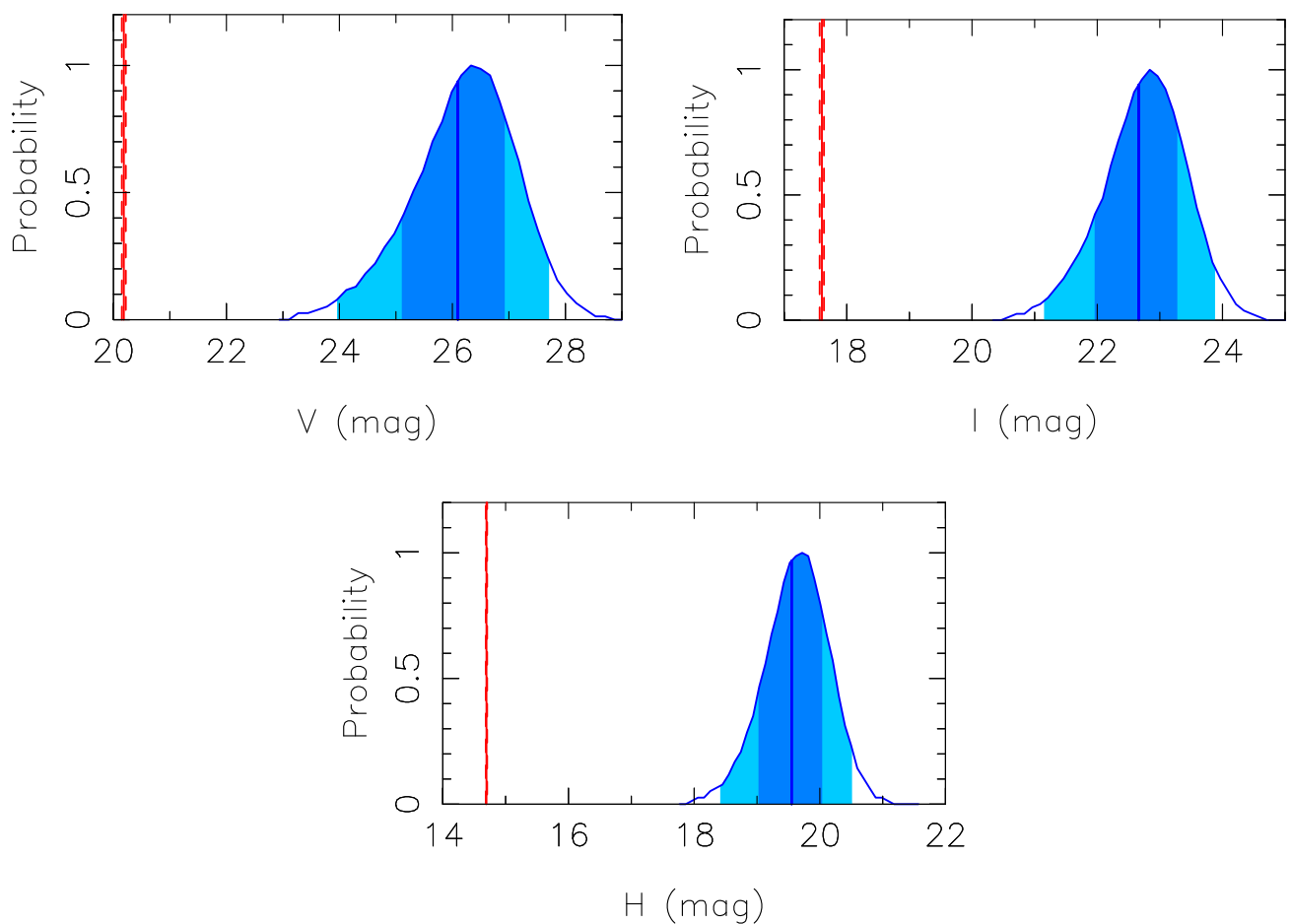

Figure 11. Probability distributions of lens brightness with extinction. The dark and light blue regions indicate the $68.3 \%$ and $95.4 \%$ confidence intervals, and the vertical blue lines indicate the median value. The red solid and dashed lines indicate the source brightness and its $1 \sigma$ errors from the light-curve fitting

Table 7 indicates that the system is composed of an early M-dwarf host $\left(M_{\text {host }}=0.56 \pm 0.07 M_{\odot}\right)$ orbited by a Saturnmass planet $\left(M_{\text {planet }}=0.41 \pm 0.05 M_{\text {Jup }}\right)$ at projected separation, $a_{\perp}=3.5 \pm 0.3 \mathrm{au}-$ that is, just over twice the snow line (assuming that this scales as $r_{\text {snow }}=2.7 \mathrm{au}\left(M / M_{\odot}\right)$ ). The lens system lies at $D_{\mathrm{L}}=5.2 \pm 0.5 \mathrm{kpc}$ (i.e., somewhat more than halfway toward the bulge). It is moving in the azimuthal direction at a speed, $v_{\mathrm{L}, l}=230 \pm 33 \mathrm{~km} \mathrm{~s}^{-1}$, that is just $1 \sigma$ above the median $\left(\widetilde{v_{l}}=195 \mathrm{~km} \mathrm{~s}^{-1}\right.$ or $\left.182 \mathrm{~km} \mathrm{~s}^{-1}\right)$ for thin and thick disk stars, and $2 \sigma$ above the median $\left(\widetilde{v_{l}}=112.3 \mathrm{~km} \mathrm{~s}^{-1}\right)$ 
Table 8

Model Stellar Velocities at $D=5.2 \pm 0.5$

\begin{tabular}{|c|c|c|c|c|c|c|}
\hline Star Component & $\begin{array}{l}\text { Velocity Component } \\
\qquad \mathrm{km} \mathrm{s}^{-1}\end{array}$ & $-2 \sigma$ & $-1 \sigma$ & $\operatorname{Median}(\widetilde{v})$ & $+1 \sigma$ & $+2 \sigma$ \\
\hline \multirow[t]{2}{*}{ Thin Disk Stars } & $v_{l}$ & 92.4 & 148.3 & 195.1 & 236.2 & 278.4 \\
\hline & $v_{b}$ & -70.4 & -30.9 & 0.8 & 30.9 & 70.2 \\
\hline Thick Disk Stars & $v_{b}$ & -127.3 & -63.9 & 1.1 & 67.5 & 127.8 \\
\hline \multirow[t]{2}{*}{ Bulge Stars } & $v_{l}$ & -12.6 & 50.8 & 112.3 & 172.7 & 231.2 \\
\hline & $v_{b}$ & -109.6 & -53.8 & -0.4 & 54.6 & 112.5 \\
\hline
\end{tabular}

for bulge stars, given in Table 8 . The lens vertical velocity $v_{\mathrm{L}, b}=64 \pm 8 \mathrm{~km} \mathrm{~s}^{-1}$ is within $1 \sigma$ above the median for thick disk stars and between $1 \sigma$ and $2 \sigma$ above the median for the thin disk and bulge stars. The thin disk, thick disk, and bulge stars comprise $80 \%, 11 \%$, and $9 \%$, respectively, of the stars that provide $t_{\mathrm{E}} \approx 37$ days events at $D_{\mathrm{L}}=5.2 \pm 0.5 \mathrm{kpc}$. Therefore, the lens system is most likely to be part of the thin or thick disk population, but a bulge lens system cannot be ruled out.

The source and lens magnitudes are also given in Table 7 and Figure 11. The lens magnitudes were calculated from the host star masses from our MCMC over light-curve models using the empirical mass-luminosity relation described by Bennett et al. (2018a), which is a combination of several different massluminosity relations for different mass ranges. For $M_{\mathrm{L}} \geqslant$ $0.66 M_{\odot}, 0.54 M_{\odot} \geqslant M_{\mathrm{L}} \geqslant 0.12 M_{\odot}$, and $0.10 M_{\odot} \geqslant M_{\mathrm{L}} \geqslant$ $0.07 M_{\odot}$, we use the relations of Henry \& McCarthy (1993), Delfosse et al. (2000), and Henry et al. (1999), respectively. In between these mass ranges, we linearly interpolate between the two relations used on the boundaries. That is, we interpolate between the Henry \& McCarthy (1993) and the Delfosse et al. (2000) relations for $0.66 M_{\odot}>M_{\mathrm{L}}>0.54 M_{\odot}$, and we interpolate between the Delfosse et al. (2000) and Henry et al. (1999) relations for $0.12 M_{\odot}>M_{\mathrm{L}}>0.10 M_{\odot}$.

The detection of the lens star in follow-up observations will be somewhat challenging because the source is a first-ascent giant only $1.3 \mathrm{mag}$ fainter than the red clump. The median predicted lens magnitude, $H_{\mathrm{L}}$, is $4.9 \mathrm{mag}$ fainter than the source, which means that it is fainter than the calibration uncertainty in $H_{\mathrm{L}}$, so we cannot expect to detect any significant excess flux at the position of the source, unless the lens is near the $2 \sigma$ upper limit on its brightness. Also, the relatively red source implies that it will be difficult to detect the lens star using the color-dependent centroid shift (Bennett et al. 2006) because the lens is likely to have a similar color to the source. The image elongation (Bennett et al. 2007) is also difficult to measure with such a high ratio between the source and lens brightnesses. Thus, the detection of the lens star will be significantly more challenging than previous cases with Keck adaptive optics (AO) imaging (Batista et al. 2015; Bhattacharya et al. 2018; Vandorou et al. 2019; Bennett et al. 2020), in which image separations of $0.62-1.47$ FWHM were measured at flux ratios of 1.46-3.15. Extrapolating from Figure 1 of Bennett et al. (2020), we estimate that the lens and source can be confidently resolved at 1.3 FWHM (i.e., 72 mas with Keck $K$ band AO). This requires waiting until 2029. In any case, they would be resolved at first AO-light on the next generation of extremely large telescopes (ELTs).

\section{Discussion}

\subsection{Eighth Spitzer-sample Planet}

In order to derive statistically robust conclusions about planets from a given microlensing sample, the events must enter the sample without regard to whether they have planets or not. ${ }^{63}$ For the Spitzer sample, this criterion gives rise to two distinct issues: (1) the events should be chosen for observations and assigned an observational cadence without regard to the presence of planets, and (2) the data-quality threshold for entering the sample should be the same for events with and without planets.

Regarding the first, Yee et al. (2015a) gave detailed prescriptions for including events under various modes of selection. However, from the present perspective the situation is relatively simple: OGLE-2017-BLG-0406 met so-called objective criteria and thus had to be observed, regardless of whether it had a planet or not. Moreover, it was observed at an "objectively determined" cadence. The only exception to this was that, as with essentially all known planetary events, OGLE-2017-BLG-0406 was observed at baseline during the 2019 season. The main motivation for this was to test for systematics, in part due to concerns raised by Koshimoto \& Bennett (2019). See, for example, Gould et al. (2020). The addition of baseline data leads to a more precise parallax measurement. Thus, while these additional data are not in themselves relevant to the present point, they are to the next one.

Second, Zhu et al. (2017) established the following dataquality condition for the statistical study of the Galactic distribution of planets: the parallax should be adequately measured, meaning that the event should only be included in the sample provided that the error in " $D_{8.3}$ " satisfies

$$
\sigma\left(D_{8.3}\right)<1.4 \mathrm{kpc} ; \quad D_{8.3} \equiv \frac{\mathrm{kpc}}{\pi_{\mathrm{rel}} / \mathrm{mas}+1 / 8.3}
$$

where $\pi_{\text {rel }}=\theta_{\mathrm{E}} \pi_{\mathrm{E}}$. However, in order that this criterion be independent of the presence of the planet, they require that the estimate of $\sigma\left(D_{8.3}\right)$ be derived from the corresponding singlelens event (with planet removed), rather than the actual event, which has added information from the planetary anomaly and (possibly) finite-source effects.

\footnotetext{
${ }^{63}$ But see Udalski et al. (2018) for an alternative planet-only based approach.
} 
Therefore, we tested whether this event meets this criterion as follows. First, we make the analogous data set as described in Ryu et al. (2017). Next, we fit the data set with a single-lens model with parallax and finite-source effects-that is, six microlensing parameters $\left(t_{o}, u_{0}, t_{\mathrm{E}}, \rho, \pi_{\mathrm{E}, N}, \pi_{\mathrm{E}, E}\right)$. Note that for this purpose we remove the 2019 Spitzer data, because these would not have been obtained if there had been no planet.

In typical cases of single-lens events (whether real or, as in this case, simulated), one does not measure $\rho$ but only obtains some (usually weak) limit $z_{0} \gg 1$, where $z_{0} \equiv u_{0} / \rho$. Thus, one must estimate $D_{8.3}$ using a Bayesian analysis (Zhu et al. 2017). In the present case, $z_{0}=1.59$. Because $z_{0}>1$, there is no caustic crossing in the single-lens event, but there is significant excess magnification at peak relative to the point-source case, which can be evaluated in the quadrupole approximation (Gould 2008) as

$$
\frac{\delta A}{A}=\frac{1-\Gamma / 5}{8 z_{0}^{2}} \rightarrow 4.5 \% \text {. }
$$

Here we have adopted $\Gamma=0.5$ as an illustrative value of the limb-darkening parameter. Therefore, given the high density and precision of the data over the peak, as well as over most of the event, we expect a very good measurement of $\rho$. In fact, we find that the single-lens light curve yields excellent constraints on both $\pi_{\mathrm{E}}$ and $\rho$, with $\sigma\left(D_{8.3}\right)=0.19 \mathrm{kpc}$ (compared to $0.26 \mathrm{kpc}$ for the actual event). Therefore, in this case, it is not necessary to conduct additional Bayesian analysis because the single-lens events satisfies the Zhu et al. (2017) criterion without it.

Hence, OGLE-2017-BLG-0406Lb becomes the eighth planet in the Spitzer-statistical sample, the others being OGLE-2014-BLG-0124Lb (Udalski et al. 2015b) OGLE2015-BLG-0966Lb (Street et al. 2016), OGLE-2016-BLG1190Lb (Ryu et al. 2017), OGLE-2016-BLG-1195Lb (Bond et al. 2017; Shvartzvald et al. 2017), OGLE-2017-BLG1140Lb (Calchi Novati et al. 2018), OGLE-2018-BLG0799Lb (W. Zang et al. 2020, in preparation), and KMT2018-BLG-0029Lb (Gould et al. 2020). In addition, there are two microlensing planets from the Spitzer bulge survey that do not enter the statistical sample, OGLE-2016-BLG-1067Lb (Calchi Novati et al. 2019) and OGLE-2018-BLG-0596Lb (Jung et al. 2019), as well as one other Spitzer microlensing planet in the Galactic disk, Kojima-1b (Nucita et al. 2018; Fukui et al. 2019; Zang et al. 2020). To our knowledge, there are two other potential Spitzer-statistical-sample planets under active investigation. The Spitzer microlensing program ended in 2019.

\subsection{ID Spitzer-"only" Parallax $\cap 1 D$ Ground-only Parallax}

Figures 2 and 3 show that the OGLE-2017-BLG-0406 parallax measurement derives from the intersection of two sets of 1D parallax contours - one from the ground-only measurement and the other from the Spitzer-"only" measurement. Gould (1999) first suggested the idea of combining 1D parallax information from Spitzer with 1D information from the ground, but after Spitzer observations of $\sim 1000$ microlensing events, this is only the second case for which the intersection of such 1D information has been demonstrated.

However, this lack of identified cases may simply reflect the fact that OGLE-2017-BLG-0406 is only the fourth microlensing event for which Spitzer-"only" and ground-only parallax contours have been shown separately. ${ }^{64}$ In two of the previous cases, KMT-2018-BLG-0029 (Gould et al. 2020) and OGLE2018-BLG-0799 (W. Zang et al. 2020, in preparation) $\pi_{\mathrm{E}}$ was basically determined by Spitzer-“only," while the much weaker ground-only information served mainly to help distinguish among degenerate solutions. In the other case, Kojima-1 (Zang et al. 2020), the ground-only microlensing data also provided relatively weak constraints that mainly helped distinguish between degenerate solutions. However, in this case, there was very precise, purely 1D information from VLTI GRAVITY interferometry (Dong et al. 2019).

It would be of interest to determine whether there are other such cases, in part to determine whether (as for OGLE-2017BLG-0406) the Spitzer-"only" and ground-only contours were consistent at the $1 \sigma$ (or perhaps $2 \sigma$ ) level. This could provide important statistical information on the frequency of systematic errors in both types of data sets. We note that the analytic Equations (5)-(7) provide a fast route to mapping Spitzer"only" contours out to arbitrarily large $\sigma$.

\subsection{Future Imaging with Adaptive Optics}

With its measured mass $M=0.56 \pm 0.07 M_{\odot}$ and distance $D_{\mathrm{L}}=5.2 \pm 0.5 \mathrm{kpc}$, the OGLE-2017-BLG-0406 host is likely to be $\sim 90$ times fainter than the microlensed source in the $H$ band, with a $2 \sigma$ range of 31-210 times fainter. According to Table 7, the lens and source are separating at $\mu_{\mathrm{rel}, \mathrm{H}}=6.1 \mathrm{mas} \mathrm{yr}^{-1}$ (i.e., just slightly larger than the geocentric proper motion). This implies a separation of $\sim 49$ mas in 2025 and $\sim 73$ mas in 2029. With current instrumentation, the Keck AO system can probably detect the lens in 2029, but with improved instrumentation (Wizinowich et al. 2019), excellent AO corrections may be possible in the $H$ or $J$ bands, allowing detection in 2025. With an ELT, it may be possible to detect it sooner. James Webb Space Telescope may be able to detect it earlier than 2025 via image elongation (Bennett et al. 2007) or the color-dependent centroid method (Bennett et al. 2006; Bhattacharya et al. 2019). Multi-orbit HST observations might be able to detect the lens by 2025 , but the source and lens are much too faint for VLT GRAVITY.

Work by Y.H. was supported by JSPS KAKENHI grant No. 17J02146. D.P.B., A.B., and C.R. were supported by NASA through grant NASA-80NSSC18K0274. Work by N.K. is supported by JSPS KAKENHI grant No. JP18J00897. Work by C.R. was supported by an appointment to the NASA Postdoctoral Program at the Goddard Space Flight Center, administered by USRA through a contract with NASA. Work by A.G. was supported by AST-1516842 from the US NSF and by JPL grant 1500811. A.G. received support from the European Research Council under the European Unions Seventh Framework Programme (FP 7) ERC grant Agreement No. [321035]. Work by C.H. was supported by the grants of the National Research Foundation of Korea (2017R1A4A1015178 and 2019R1A2C2085965). The MOA project is supported by JSPS KAKENHI grant No. JSPS24253004, JSPS26247023, JSPS23340064, JSPS15H00781, JP16H06287, 17H02871, and

\footnotetext{
64 Jung et al. (2019) separately analyzed the Spitzer-"only" and ground-only $\boldsymbol{\pi}_{\mathrm{E}}$ measurements for OGLE-2018-BLG-0596, but they only showed contours for the former. We have checked, using an analog of Figures 2 and 3 , that the $1 \sigma$ parallax contours overlap for the preferred solution $\left(s<1, u_{0}<0\right)$. This is a useful check on systematics for the case of that event. However, because the Spitzer-"only" arc and the ground-only ellipse are essentially tangent at the point of intersection, this is not a case of combining two 1D measurements to form a $2 \mathrm{D}$ measurement.
} 
19KK0082. The OGLE project has received funding from the National Science Centre, Poland, grant MAESTRO 2014/14/ A/ST9/00121 to AU. This research has made use of the KMTNet system operated by the Korea Astronomy and Space Science Institute (KASI), and the data were obtained at three host sites of CTIO in Chile, SAAO in South Africa, and SSO in Australia. Y.T. acknowledges the support of DFG priority program SPP 1992 "Exploring the Diversity of Extrasolar Planets" (WA 1047/11-1).

\section{Appendix \\ Investigation of Trends in the Spitzer Residuals}

The Spitzer residuals to the upper panel of Figure 1 show a low-amplitude (compared to the overall 2017-2019 flux variation) "wave" that appears to be driven by six systematically high points at the end of the 2017 data. If we remove these six points from the fit, then $\chi^{2}$ for the Spitzer data improves by $\Delta \chi^{2}=13.5$. We find numerically that for a Gaussian series of 28 points, the probability that there are six consecutive points with total contribution $\chi^{2}>13.5$, and all above or all below the model, is $1.7 \%$. For a test made a priori, this would be fairly compelling evidence of systematic effects. For an a posteriori test that is constructed to match visually identified features in the data, it is less so. Nevertheless, this test motivates us to check the impact on the final results of including versus excluding these six points.
We therefore repeat the analysis of Section 5.2 after first removing the six points and also re-renormalize the error bars by a further factor of 0.78 in order to enforce $\chi^{2} /$ dof $=1$. Figure A1 shows the resulting analog of Figure 2. The main change is that the Spitzer-"only" minimum slides "along the arc" toward its center. However, the point of intersection with the ground-only solution barely changes, with the net result being that the combined solution (right panel) barely changes.

This relative insensitivity of the final result to the details of the late-time 2017 data can be understood within the framework of the Gould (2019) "osculating circle" analysis. Each of the earliest 2017 points (together with the 2019 baseline and the color-color constraint) yields a circle in the $\pi_{\mathrm{E}}$ plane. These osculating circles differ slightly in radius (and center), and so their overlap produces an arc. Late-time data can fill in the details of this arc, but these details are largely irrelevant because, to zeroth order, it is only the point where the arc crosses the major axis of the ground-only error ellipse that defines the solution. At first order, the position of the minimum along the Spitzer-"only" arc plays some role because the ground-only contours have a finite width. However, as this width is small, the best fit can only be moved from one side to the other of this narrow range.

Tables A1 and A2 give the microlensing and physical parameters for the $\mathrm{W}+$ and $\mathrm{W}-$ solutions. They can be directly compared to Tables 4 and 7 . As would be predicted from the analysis of Figure A1, the physical parameters change by very

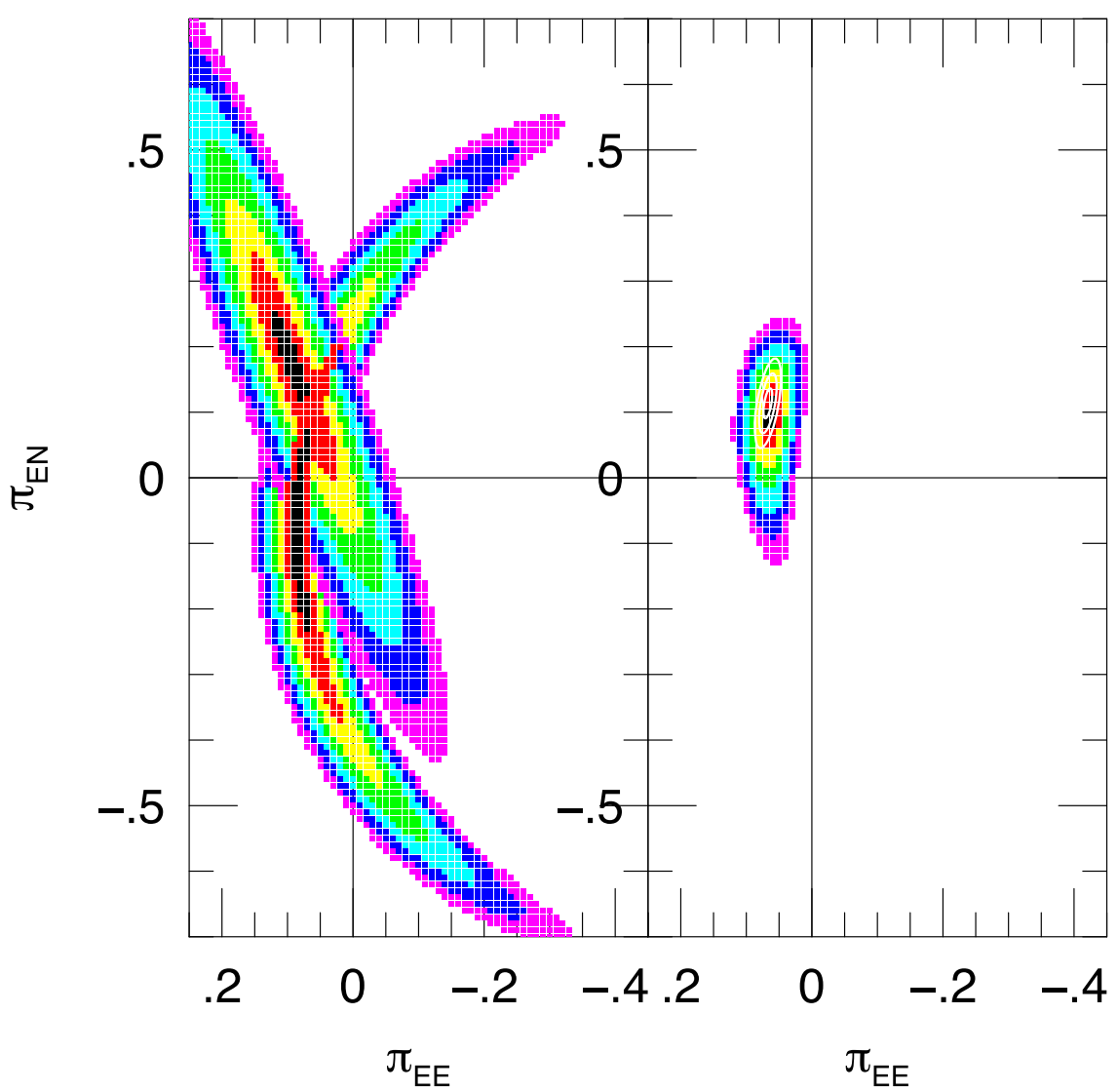

Figure A1. OGLE-2017-BLG-0406 parallax contours for the W+ solution after the removal of the last six Spitzer data points from 2017. Hence, the elliptical contours from the ground-only fit in the left panel are identical to those of Figure 2. The Spitzer arc looks qualitatively similar, but there is a single minimum near $\pi_{\mathrm{E}, N} \sim 0$ rather than two weak, roughly symmetric minima at $\pm \pi_{\mathrm{E}, N}$. Nevertheless, in both cases, the two sets of contours overlap at $1 \sigma$. The colored contours at the right show the product of the two sets of likelihood contours from the left. The white contours are from the full fit to all the data (i.e., they are the same as in Figure 2). Thus comparison of the white and colored contours shows that the solution changes by $<1 \sigma$. 
Table A1

Wide Models for Ground+Spitzer Data with 6 pts Removed

\begin{tabular}{lcc}
\hline \hline Parameters & Wide $(+,+)$ & Wide $(-,+)$ \\
\hline$t_{0}\left(\mathrm{HJD}^{\prime}\right)$ & $7908.812 \pm 0.001$ & $7908.813 \pm 0.001$ \\
$u_{0}\left(10^{-3}\right)$ & $9.298 \pm 0.028$ & $-9.281 \pm 0.028$ \\
$t_{\mathrm{E}}($ days $)$ & $37.069 \pm 0.086$ & $37.133 \pm 0.084$ \\
$s$ & $1.128 \pm 0.001$ & $1.128 \pm 0.001$ \\
$q\left(10^{-4}\right)$ & $6.924 \pm 0.091$ & $6.970 \pm 0.090$ \\
$\alpha(\mathrm{rad})$ & $0.993 \pm 0.001$ & $-0.993 \pm 0.002$ \\
$\rho\left(10^{-3}\right)$ & $5.861 \pm 0.024$ & $5.843 \pm 0.025$ \\
$\pi_{\mathrm{E}, N}$ & $0.117(0.101) \pm 0.023$ & $0.120(0.105) \pm 0.024$ \\
$\pi_{\mathrm{E}, E}$ & $0.063(0.066) \pm 0.007$ & $0.065(0.067) \pm 0.007$ \\
$\pi_{\mathrm{E}}$ & $0.133(0.121) \pm 0.017$ & $0.136(0.126) \pm 0.018$ \\
$\phi_{\pi}$ & $0.495(0.593) \pm 0.143$ & $0.499(0.584) \pm 0.141$ \\
$f_{S}($ OGLE $)$ & $1.461 \pm 0.004$ & $1.459 \pm 0.004$ \\
$f_{B}($ OGLE $)$ & $0.103 \pm 0.004$ & $0.105 \pm 0.004$ \\
$f_{S}($ Spitzer $)$ & $11.297 \pm 0.175$ & $11.265 \pm 0.182$ \\
$f_{B}($ Spitzer $)$ & $-2.841 \pm 0.176$ & $-2.808 \pm 0.183$ \\
$t_{\text {ast }}($ days $)$ & $0.217 \pm 0.001$ & $0.217 \pm 0.001$ \\
\hline
\end{tabular}

Note. Mean values from the MCMC are shown in parentheses. All other values are from the best-fit model. $\pi_{\mathrm{E}}, \phi_{\pi}$, and $t_{\text {ast }}$ are derived quantities and are not fitted independently. All fluxes are on an 18th mag scale (e.g., $I_{s}=18-2.5 \log \left(f_{s}\right)$ ).

Table A2

Physical Parameters

\begin{tabular}{lcc}
\hline \hline Parameter & Units & Values \\
\hline$M_{\text {host }}$ & $M_{\odot}$ & $0.60 \pm 0.09$ \\
$M_{\text {planet }}$ & $M_{\text {Jup }}$ & $0.44 \pm 0.06$ \\
$D_{\mathrm{L}}$ & $\mathrm{kpc}^{-1}$ & $5.10 \pm 0.39$ \\
$\mu_{\text {rel,H,N }}$ & mas yr $^{-1}$ & $4.82 \pm 0.52$ \\
$\mu_{\text {rel,H,E }}$ & mas yr $^{-1}$ & $3.64 \pm 0.61$ \\
$a_{\perp}$ & au & $3.40 \pm 0.26$ \\
\hline
\end{tabular}

little: $\leqslant 0.5 \sigma$ for the masses and by less than half that for the distances and velocities.

The causes of the systematic trends in the Spitzer data are not fully understood. Gould et al. (2020) found trends of a generally similar form but of much greater amplitude relative to the observed Spitzer flux variation, in their analysis of KMT2018-BLG-0029. They argued that these were most likely due to the effect of normal field rotation during the Spitzer observing window combined with the poorly determined positions of several nearby stars that were many magnitudes brighter than the source. They thus argued that the most robust delta-flux measurements in the light curve were those between observations early in the 2018 and early in the 2019 seasons, which all had similar field angle.

In the present case, there are no such bright contaminating nearby stars. And correspondingly, the observed trends are much weaker. Hence, perhaps there is some similar effect from the wings of more distant stars. In any case, the net effect of eliminating the last six points from 2017 is that all remaining data are from the beginning of the observing window (first 9.3 days of 2017 and first 7.0 days of 2019), when we expect the effects of field rotation to be minimized.

We infer that the radius of the osculating circles, which is the aspect of the Spitzer parallax measurement that primarily contributes to the final $\pi_{\mathrm{E}}$ measurement, is the least subject to systematic effects, because it derives directly from the comparison of early-2017 with early-2019 data, which are at the same field orientation. That is, the narrow (best statistically determined) direction of the Spitzer contours-namely, the radial coordinate defined by the circular arc-is also the most robust from the standpoint of systematics. Recall from Section 5.2 that the same was true of the ground-based contours: the narrow (best statistically determined) direction was also the more robust from the standpoint of systematic errors.

Because the case for removing the final six points from 2017 is not compelling and also because doing so changes the estimates of the physical parameters by substantially less than $1 \sigma$, we report the determinations from the full data set as our results. However, for completeness, we also list the results from fits with these six points removed in Tables A1 and A2.

\section{ORCID iDs}

David P. Bennett (1) https://orcid.org/0000-0001-8043-8413 Yoon-Hyun Ryu (iD https://orcid.org/0000-0001-9823-2907

Naoki Koshimoto (iD https://orcid.org/0000-0003-2302-9562 Andrzej Udalski (iD https://orcid.org/0000-0001-5207-5619 Jennifer C. Yee (i) https://orcid.org/0000-0001-9481-7123 Yossi Shvartzvald (i) https://orcid.org/0000-0003-1525-5041 Richard K. Barry (iD https://orcid.org/0000-0003-4916-0892 Yoshitaka Itow (i) https://orcid.org/0000-0002-8198-1968 Iona Kondo (ib https://orcid.org/0000-0002-3401-1029 Taro Matsuo (iD https://orcid.org/0000-0001-7694-5885 Shota Miyazaki (i) https://orcid.org/0000-0001-9818-1513 Clément Ranc (i) https://orcid.org/0000-0003-2388-4534 Nicholas J. Rattenbury (iD https://orcid.org/0000-00015069-319X

Daisuke Suzuki (iD https://orcid.org/0000-0002-5843-9433

J. Skowron (iD https://orcid.org/0000-0002-2335-1730

P. Pietrukowicz (i) https://orcid.org/0000-0002-2339-5899

K. Ulaczyk (iD https://orcid.org/0000-0001-6364-408X

Sun-Ju Chung (1) https://orcid.org/0000-0001-6285-4528

Cheongho Han (i) https://orcid.org/0000-0002-2641-9964

Kyu-Ha Hwang (iD https://orcid.org/0000-0002-9241-4117

In-Gu Shin (D) https://orcid.org/0000-0002-4355-9838

Weicheng Zang (i) https://orcid.org/0000-0001-6000-3463

Sean Carey (10) https://orcid.org/0000-0002-0221-6871

Calen B. Henderson (iD https://orcid.org/0000-00018877-9060

Etienne Bachelet (ib https://orcid.org/0000-0002-6578-5078 Rachel A. Street (iD https://orcid.org/0000-0001-6279-0552 Thiam-Guan Tan (i) https://orcid.org/0000-0001-5603-6895

V. Bozza (iD https://orcid.org/0000-0003-4590-0136

M. Rabus (iD https://orcid.org/0000-0003-2935-7196

L. Mancini (i) https://orcid.org/0000-0002-9428-8732

\section{References}

Alard, C., \& Lupton, R. H. 1998, ApJ, 503, 325

Albrow, M. D., Beaulieu, J.-P., Birch, P., et al. 1998, ApJ, 509, 697

Albrow, M. D., Horne, K., Bramich, D. M., et al. 2009, MNRAS, 397, 2099 Alcock, C., Allsman, R., Alves, D., et al. 1995, ApJ, 454L, 125

Batista, V., Beaulieu, J.-P., Bennett, D. P., et al. 2015, ApJ, 808, 170

Beaulieu, J.-P., Batista, V., Bennett, D. P., et al. 2018, AJ, 155, 78 Bennett, D. P. 2010, ApJ, 716, 1408

Bennett, D. P., Anderson, J., \& Bond, I. A. 2006, ApJ, 647, L171

Bennett, D. P., Anderson, J., \& Gaudi, S. 2007, ApJ, 660, 781

Bennett, D. P., Batista, V., Bond, I. A., et al. 2014, ApJ, 785, 155 
Bennett, D. P., Bhattacharya, A., Anderson, J., et al. 2015, ApJ, 808, 169 Bennett, D. P., Bhattacharya, A., Beaulieu, J. P., et al. 2020, AJ, 159, 68 Bennett, D. P., Bond, I. A., Udalski, A., et al. 2008, ApJ, 684, 663 Bennett, D. P., \& Rhie, S. H. 1996, ApJ, 472, 660

Bennett, D. P., \& Rhie, S. H. 2002, ApJ, 574, 985

Bennett, D. P., Rhie, S. H., Nikolaev, S., et al. 2010, ApJ, 713, 837

Bennett, D. P., Rhie, S. H., Udalski, A., et al. 2016, AJ, 152, 125

Bennett, D. P., Udalski, A., Bond, I. A., et al. 2018a, AJ, 156, 113

Bennett, D. P., Udalski, A., Han, C., et al. 2018b, AJ, 155, 141

Bensy, T., Yee, J. C., Felzing, S., et al. 2013, A\&A, 549, A147

Bessell, M. S., \& Brett, J. M. 1988, PASP, 100, 1134

Bhattacharya, A., Anderson, J., Beaulieu, J. P., et al. 2019, Host Mass and

Distance Dependence of Wide Orbit Planets with Near Simultaneous HST and Keck AO Observations, HST Proposal, 15690

Bhattacharya, A., Beaulieu, J. P., Bennett, D. P., et al. 2018, AJ, 156, 289

Bhattacharya, A., Bennett, D. P., Anderson, J., et al. 2017, AJ, 154, 59

Bond, I. A., Abe, F., Dodd, R. J., et al. 2001, MNRAS, 327, 868

Bond, I. A., Bennett, D. P., Sumi, T., et al. 2017, MNRAS, 469, 2434

Borucki, W. J., Koch, D. G., Basri, G., et al. 2011, ApJ, 736, 19

Boyajian, T. S., van Belle, G., \& von Braun, K. 2014, AJ, 147, 47

Bramich, D. M. 2008, MNRAS, 386, L77

Butler, R. P., Wright, J. T., Marcy, G. W., et al. 2006, ApJ, 646, 505

Calchi Novati, S., Gould, A., Yee, J. C., et al. 2015, ApJ, 814, 92

Calchi Novati, S., Skowron, J., Jung, Y. K., et al. 2018, AJ, 155, 261

Calchi Novati, S., Suzuki, D., Udalski, A., et al. 2019, AJ, 157, 121

Cardelli, J. A., Clayton, G. C., \& Mathis, J. S. 1989, ApJ, 345, 245

Carpenter, J. M. 2001, AJ, 121, 2851

Cassan, A., Kubas, D., Beaulieu, J.-P., et al. 2012, Natur, 481, 167

Chung, S.-J., Gould, A., Skowron, J., et al. 2019, AJ, 179, 13

Chung, S.-J., Han, C., Park, B.-G., et al. 2005, ApJ, 630, 535

Claret, A. 2000, A\&A, 363, 1081

Delfosse, X., Forveille, T., Ségransan, D., et al. 2000, A\&A, 364, 217

Dominik, M. 1999, A\&A, 349, 108

Dong, S., Gould, A., Udalski, A., et al. 2009, ApJ, 695, 970

Dong, S., Mérand, A. M., Delplancke-Strobele, F., et al. 2019, ApJ, 871, 70

Fukui, A., Suzuki, D., Koshimoto, N., et al. 2019, AJ, 158, 206

Gaia Collaboration, Katz, D., Antoja, T., et al. 2018, A\&A, 616, A11

Gaudi, B. S. 1998, ApJ, 506, 533

Gaudi, B. S. 2012, ARA\&A, 50, 411

Gaudi, B. S., Bennett, D. P., Udalski, A., et al. 2008, Sci, 319, 927

González Hernández, J. I., Bonifacio, P., et al. 2009, A\&A, 497, 497

Gould, A. 1992, ApJ, 392, 442

Gould, A. 1994, ApJL, 421, L75

Gould, A. 1999, ApJ, 514, 869

Gould, A. 2000, ApJ, 5442, 785

Gould, A. 2004, ApJL, 606, 319

Gould, A. 2008, ApJ, 681, 1593

Gould, A. 2019, JKAS, 52, 121

Gould, A., Dong, S., Gaudi, B. S., et al. 2010, ApJ, 720, 1073

Gould, A., \& Loeb, A. 1992, ApJ, 396, 104

Gould, A., Miralda-Escudé, J., \& Bahcall, J. N. 1994, ApJ, 423, L105

Gould, A., Ryu, Y.-H., Calchi Novati, S., et al. 2020, JKAS, 53, 9

Gould, A., Udalski, A., Shin, I.-G., et al. 2014, Sci, 345, 46

Griest, K., \& Safizadeh, N. 1998, ApJ, 500, 37

Henry, T. J., Franz, O. G., Wasserman, L. H., et al. 1999, ApJ, 512, 864

Henry, T. J., \& McCarthy, D. W., Jr. 1993, AJ, 106, 773

Ida, S., \& Lin, D. N. C. 2004, ApJ, 604, 388

Ida, S., \& Lin, D. N. C. 2005, ApJ, 625, 1045

Jung, Y., Gould, A., Zang, W., et al. 2018, AJ, 157, 72

Jung, Y. K., Gould, A., Udalski, A., et al. 2019, AJ, 158, 28

Kervella, P., Thev́enin, F., Di Folco, E., \& Seǵransan, D. 2004, A\&A, 426, 297

Kim, D.-J., Kim, H.-W., Hwang, K.-H., et al. 2018, AJ, 155, 76
Kim, S.-L., Lee, C.-U., Park, B.-G., et al. 2016, JKAS, 49, 37

Koshimoto, N., \& Bennett, D. P. 2019, arXiv:1905.05794

Koshimoto, N., Bennett, D. P., \& Suzuki, D. 2020, AJ, 159, 268

Koshimoto, N., Shvartzvald, Y., Bennett, D. P., et al. 2017, AJ, 154, 3

Lissauer, J. J., Hubickyj, O., Da, Angelo, G., \& Bodenheimer, P. 2009, Icar, 199, 338

Mróz, P., Poleski, R., Han, C., et al. 2020, AJ, 159, 262

Mróz, P., Ryu, Y.-H., Skowron, J., et al. 2018, AJ, 155, 121

Mróz, P., Udalski, A., Bennett, D. P., et al. 2019, A\&A, 622, 201

Mróz, P., Udalski, A., Skowron, J., et al. 2017, Natur, 548, 183

Muraki, Y., Han, C., Bennett, D. P., et al. 2011, ApJ, 741, 22

Nagayama, T., Nagashima, C., Nakajima, Y., et al. 2003, Proc. SPIE, 4841, 459

Nataf, D. M., Gonzalez, O. A., Casagrande, L., et al. 2016, MNRAS, 456, 2692

Nataf, D. M., Gould, A., Fouqué, P., et al. 2013, ApJ, 769, 88

Nayakshin, S., Dipierro, G., \& Szulágyi, J. 2019, MNRAS, 488, L12

Nishiyama, S., Nagata, T., Tamura, M., et al. 2008, ApJ, 680, 1174

Nishiyama, S., Tamura, M., Hatano, H., et al. 2009, ApJ, 696, 1407

Nucita, A. A., Licchelli, D., De Paolis, F., et al. 2018, MNRAS, 476, 2962

Penny, M., Henderson, C. B., \& Clanton, C. , 2016, ApJ, 830, 150

Pollack, J. B., Hubickyj, O., Bodenheimer, P., et al. 1996, Icar, 124, 62

Refsdal, S. 1966, MNRAS, 134, 315

Rhie, S. H., Becker, A. C., Bennett, D. P., et al. 1999, ApJ, 522, 1037

Ryu, Y.-H., Udalski, A., Yee, J. C., et al. 2017, AJ, 155, 40

Sako, T., Sekiguchi, T., Sasaki, M., et al. 2008, ExA, 22, 51

Schechter, P. L., Mateo, M., \& Saha, A. 1993, PASP, 105, 1342

Sharma, S., Bland-Hawthorn, J., Binney, J., et al. 2014, ApJ, 793, 51

Shvartzvald, Y., Maoz, D., Udalski, A., et al. 2016, MNRAS, 457, 4089

Shvartzvald, Y., Yee, J. C., Calchi Novati, S., et al. 2017, ApJL, 840, L3

Shvartzvald, Y., Yee, J. C., Skowron, J., et al. 2019, AJ, 157, 106

Skowron, J., Shin, I.-G., Udalski, A., et al. 2015, ApJ, 804, 33

Skowron, J., Udalski, A., Kozĺowski, S., et al. 2016a, AcA, 66, 1

Skowron, J., Udalski, A., Poleski, R., et al. 2016b, ApJ, 820, 4

Smith, M., Mao, S., \& Paczyński, B. 2003, MNRAS, 339, 925

Spergel, D., Gehrels, N., Baltay, D., et al. 2015, arXiv:1503.03757

Street, R., Udalski, A., Calchi Novati, S., et al. 2016, ApJ, 829, 93

Sumi, T., Bennett, D. P., Bond, I. A., et al. 2010, ApJ, 710, 1641

Sumi, T., Kamiya, K., Bennett, D. P., et al. 2011, Natur, 473, 349

Sumi, T., Udalski, A., Bennett, D. P., et al. 2016, ApJ, 825, 112

Sumi, T., Wu, X., Udalski, A., et al. 2004, MNRAS, 348, 1439

Suzuki, D., Bennett, D. P., Bond, S. T., et al. 2016, ApJ, 833, 145

Suzuki, D., Bennett, D. P., Ida, S., et al. 2018, ApJL, 869, 34

Szulágyi, J., Morbidelli, A., Crida, A., et al. 2014, ApJ, 782, 65

Tomaney, A. B., \& Crotts, A. P. S. 1996, AJ, 112, 287

Tsapras, Y. 2018, Geosc, 8, 365

Udalski, A. 2003, AcA, 53, 291

Udalski, A., Jaroszyński, M., Paczyński, B., et al. 2005, ApJ, 628, 109

Udalski, A., Ryu, Y.-H., Sajadian, S., et al. 2018, AcA, 68, 1

Udalski, A., Szymański, M. K., \& Szymański, G. 2015a, AcA, 65, 1

Udalski, A., Yee, J. C., Gould, A., et al. 2015b, ApJ, 799, 237

Vandorou, A., Bennett, D. P., Beaulieu, J.-P., et al. 2019, arXiv:1909.04444

Verde, L., Peiris, H. V., \& Spergel, D. N. 2003, ApJS, 148, 195

Wizinowich, P., Chin, J., Casey, K., et al. 2019, Adaptive Optics for Extremely Large Telescopes (AO4ELT6), http://ao4elt6.copl.ulaval.ca/proceedings / 401-fDmd-211.pdf

Woźniak, P. R. 2000, AcA, 50, 421

Yee, J. C., Gould, A., \& Beichman, C. , 2015a, ApJ, 810, 155

Yee, J. C., Udalski, A., Calchi Novati, S., et al. 2015b, ApJ, 802, 76

Yoo, J., Depoy, D. L., Gal-Yam, A., et al. 2004, ApJ, 603, 139

Zang, W., Dong, S., Gould, A., et al. 2020, arXiv:1912.00038

Zhu, W., Udalski, A., Calchi Novati, S., et al. 2017, AJ, 154, 210 